\title{
The nuclear ubiquitin ligase adaptor SPOP is a conserved regulator of C9orf72 dipeptide toxicity
}

Carley Snoznik ${ }^{1}$, Valentina Medvedeva ${ }^{3}$, Jelena Mojsilovic-Petrovic ${ }^{3}$, Paige Rudich $^{1,2}$, James Oosten ${ }^{1}$, Robert G. Kalb* ${ }^{3}$, Todd Lamitina*,1,2,4

1Division of Child Neurology, Department of Pediatrics, Children's Hospital of Pittsburgh, University of Pittsburgh Medical Center, Pittsburgh, PA, USA

${ }^{2}$ Graduate Program in Cell Biology and Molecular Physiology, University of Pittsburgh Medical Center

${ }^{3}$ Department of Neurology, Feinberg School of Medicine, Northwestern University

${ }^{4}$ Department of Cell Biology, University of Pittsburgh School of Medicine

*Co-corresponding authors. Address correspondence to: Todd Lamitina, Ph.D., Children's Hospital of Pittsburgh of the University of Pittsburgh Medical Center, Departments of Pediatrics and Cell Biology, 4401 Penn Avenue, Rangos 7122, Pittsburgh, PA 15224, phone - (412) 692-9437; fax - (412) 641-1844; email st152@pitt.edu; Robert G. Kalb, M.D. Department of Neurology, Feinberg School of Medicine, Northwestern University, 303 East Chicago Avenue, Chicago, IL 60611 phone (312) 503 5358; fax (302) 503 3950; email -

Robert.kalb1@northwestern.edu 


\section{Abstract}

A hexanucleotide repeat expansion in the C9orf72 gene is the most common cause of inherited amyotrophic lateral sclerosis (ALS) and frontotemporal dementia (FTD). Unconventional translation of the C9orf72 repeat produces dipeptide repeat proteins (DPRs). Previously, we showed that the DPRs (PR)50 and (GR)50 are highly toxic when expressed in $C$. elegans and this toxicity depends on nuclear localization of the DPR. In an unbiased genomewide RNAi screen for suppressors of (PR)50 toxicity, we identified 12 genes that consistently suppressed either the developmental arrest and/or paralysis phenotype evoked by (PR)50 expression. All of these genes have vertebrate homologs and 7/12 contain predicted nuclear localization signals. One of these genes was spop-1, the $C$. elegans homolog of SPOP, a nuclear localized E3 ubiquitin ligase adaptor only found in metazoans. SPOP is also required for (GR)50 toxicity and functions in a genetic pathway that includes cul-3, which is the canonical E3 ligase partner for SPOP. Genetic or pharmacological inhibition of SPOP in mammalian primary spinal cord motor neurons suppressed DPR toxicity without affecting DPR expression levels. Finally, we find that genetic inhibition of bet-1, the $C$. elegans homolog of the known SPOP ubiquitination targets BRD2/3/4, suppresses the protective effect of SPOP mutations.

Together, these data suggest a model in which SPOP promotes the DPRdependent ubiquitination and degradation of BRD proteins. We speculate the pharmacological manipulation of this pathway, which is currently underway for 
bioRxiv preprint doi: https://doi.org/10.1101/2021.03.09.434618; this version posted March 10, 2021. The copyright holder for this preprint

(which was not certified by peer review) is the author/funder, who has granted bioRxiv a license to display the preprint in perpetuity. It is made available under aCC-BY-NC-ND 4.0 International license.

multiple cancer subtypes, could also represent a novel entry point for therapeutic intervention to treat C9 FTD/ALS. 


\section{Significance statement}

The G4C2 repeat expansion in the C9orf72 gene is a major cause of FrontoTemporal Dementia (FTD) and Amyotrophic Lateral Sclerosis (ALS). Unusual translation of the repeat sequence produces two highly toxic dipeptide repeat proteins, PRx and GRx, which accumulate in the brain tissue of individuals with these diseases. Here, we show that PR and GR toxicity in both $C$. elegans and mammalian neurons depends on the E3 ubiquitin ligase adaptor SPOP. SPOP acts through the bromodomain protein BET-1 to mediate dipeptide toxicity. SPOP inhibitors, which are currently being developed to treat SPOP-dependent renal cancer, also protect neurons against DPR toxicity. Our findings identify a highly conserved and 'druggable' pathway that may represent a new strategy for treating these currently incurable diseases. 


\section{Introduction}

Mutations in C9ORF72 are the most common monogenic cause of ALS and FTD (the most common non-Alzheimer's adult dementia) $(1,2)$. The mutation is an expansion of the microsatellite repeat, 5'-GGGGCC-3' in the intron between exons $1 \mathrm{~A}$ and $1 \mathrm{~B}$. In unaffected individuals, the hexanucleotide repeat is reiterated $5-15$ times, while in ALS/FTD patients, the hexanucleotide repeat expansion (HRE) occurs hundreds or even thousands of times. Three nonmutually exclusive C9 pathophysiological mechanisms have been proposed: 1) reduced abundance of the native C9 protein, 2) C9 pre-mRNA containing the HRE adopts an imperfect hairpin structure that sequesters RNA binding proteins, and 3) the generation of dipeptide repeat proteins (DPRs) by non-AUGdependent translation (RANT) (3) of the HRE C9 pre-mRNA (4).

RANT of the sense $\mathrm{G}_{4} \mathrm{C}_{2}$ sequence, as well as a disease-associated antisense $\mathrm{G}_{2} \mathrm{C}_{4}$ sequence, leads to the production of five DPRs - Gly-Ala (GA), Gly-Pro (GP), Gly-Arg (GR), Pro-Ala (PA), and Pro-Arg (PR) (5). At autopsy, each of these DPRs is detected immunohistologically in C9 FTD and ALS patients but not non-C9 FTD or ALS patients. While DPR sites of expression and overall levels are highly variable, they are significantly more abundant within frontal and temporal lobe regions affected in FTD (6), suggesting a significant pathological role in this disease.

Of the five DPRs, two - Gly-Arg (GR) and Pro-Arg (PR) - are toxic in multiple cellular and animal model systems, although Gly-Ala (GA) can also be toxic in some, but not all, settings (7-9). Pathophysiological processes linked to 
DPRs include: 1) perturbation of nucleocytoplasmic shuttling $(10,11), 2)$ impaired protein translation (12), 3) Inhibition of proteasome function $(13,14), 4)$ defects in U2 snSNP-dependent splicing (15), 5) compromised mitochondrial function (16), and 6) perturbation of membraneless organelle dynamics $(17,18)$. In all of these studies, the key experimental approach involved the non-RANT expression of codon-varied DPRs, which allows experimental separation of G4C2 RNA toxicity from individual DPR toxicity. It is believed that these codon-varied DPR models accelerate the appearance of phenotypes that ordinarily emerge over decades in human C9 patients $(7,12,17-25)$.

Here we describe an unbiased genetic screen for DPR modifiers in $C$. elegans. The genes identified in this screen included many previously identified DPR modifiers, as well as several novel genes. One novel (PR)50 suppressor

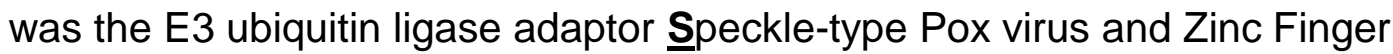
( $\underline{\text { POZ) }}$ domain Protein, or SPOP, which is a common genetic cause of prostate, endometrial, and renal cancer (26) but has never been linked to neurodegenerative disease. CRISPR-based introduction of SPOP missense alleles frequently found in prostate cancer also provided protection against (PR)50 toxicity. SPOP inhibition is a conserved mechanism to protect against DPR toxicity since either SPOP knockdown or an SPOP inhibitor in rat primary neurons also provided protection against DPR-induced neuron death. Finally, we find that the protective effects of SPOP inhibition depends on the bromodomaincontaining protein and known SPOP substrate bet-1. 


\section{Results}

\section{A genome-wide RNAi screen for suppressors of DPR toxicity in C. elegans identifies known and novel conserved genes}

Expression of C9orf72 DPRs (PR)50 and (GR)50 are toxic and cause cellular and organismal toxicity in multiple systems $(7,9,18,19,21,27)$. Expression of these DPRs in $C$. elegans muscles cells leads to a completely penetrant larval developmental arrest and paralysis phenotype (20). To identify genes required for this phenotype, we performed a genome-wide RNAi screen for suppressors of (PR)50 induced developmental arrest and/or paralysis. We screened 15,865 individual gene knockdowns and identified 391 initial (PR)50 suppressors. Each hit was rescreened in six independent trials. 12/391 hits showed suppression of the (PR)50 growth arrest in at least 4/6 of these trials (Table 1, Fig. S1). 83\% of gene knockdowns (10/12) also suppressed toxicity caused by (GR)50 in at least 1/2 age-dependent paralysis assays (Table 1, Fig. S2). Since loss of these genes suppresses toxicity, these genes are necessary for DPR toxicity. The genes identified as (PR)50 suppressors fell into three broad functional classes; 1) Nuclear transport and RNA binding, 2) Ubiquitinmediated protein degradation, and 3) chromatin regulation (Table 1). $50 \%$ $(6 / 12)$ of these genes encode homologs of genes previously identified as modifiers of or interactors with (PR)50 or (GR)50 in yeast, fly, or mammalian cells $(9,18,19,21)$, suggesting (PR)50 engages similar pathophysiological mechanisms in C. elegans as it does in other systems. $75 \%(9 / 12)$ of (PR)50 
suppressors are conserved from yeast to humans, while $25 \%(3 / 12)$ genes are absent from yeast but present in metazoan genomes. None of the genes are specific to $C$. elegans. Only one gene, ergo-1, appears to act via transgene suppression since knockdown reduces both GFP and RFP expression by $>50 \%$ from a transgene expression control strain (Fig. S3), which is consistent with previous reports of ergo-1(RNAi) acting as a transgene suppressor (28). $75 \%$ of genes (9/12) contain either a predicted nuclear localization signal or experimental evidence for nuclear localization, suggesting the encoded protein is localized to the nucleus where (PR)50 is required for toxicity (Table 1) (20). 75\% of genes (9/12) are annotated as having 'lethal' and/or 'sterile' phenotypes, suggesting that traditional forward genetic screening for DPR suppressors would not have isolated these genes since these screens usually require mutants to be viable and fertile in order to be identified (Table 1). Therefore, our RNAi screen identified highly conserved and essential genes required for (PR)50 toxicity, many of which are likely to be localized to the nucleus, where (PR)50 is also required for toxicity.

We obtained previously characterized mutant alleles that were viable and fertile for several of the genes identified in our screen. To validate our RNAi results, we crossed two of these mutants, met-2(n4256) and rsp-6(ok798), into the (PR)50 background. As we found for RNAi knockdown, both loss-of-function mutants suppressed the developmental arrest phenotype and the postdevelopmental age-dependent paralysis phenotype caused by (PR)50 (Fig. 1C). We examined (PR)50 localization in each of these mutants and found that 
nuclear localization of the DPR was disrupted. In either mutant background, (PR)50-GFP was distributed throughout the non-nuclear compartments and was largely absent from either the nucleus or nucleolus (Fig. 1D). These data provide independent validation of the hits derived from our RNAi screen and suggest that the mechanism of suppression for some genes is associated with re-localization of (PR)50 out of the nucleus.

\section{Loss-of-function mutations in the E3 ubiquitin ligase adaptor SPOP suppress (PR)50 and (GR)50 toxicity}

In addition to several previously identified DPR modulators ( $r s p-6 / S R S F 3$, ima-3/KPNA3, ztf-4/hnRNPC, gyf-1/GIGYF, rpn-12/PSMD8, utx-1/UTX/UTY), our screen also identified several new genes associated with regulation of ubiquitin proteasome activity. One newly identified gene was spop-1, the $C$. elegans homolog of human speckled-type POZ domain containing protein (SPOP) (Fig. 2A,B). spop-1(RNAi) robustly suppressed the age-dependent toxicity of both (PR)50 and (GR)50 (Fig. 2C,D). We obtained or generated via CRISPR/Cas9 mutations in spop-1 (gk630214, dr28) and crossed them into (PR)50 and (GR)50 expressing C. elegans. spop-1(gk630214) is a nonsense mutation early in the spop-1 coding sequence that truncates the protein within the MATH domain and is therefore likely a null allele (Fig. 2A). spop-1(dr28) is a CRISPR engineered 82bp deletion within exon 2 that deletes the start codon for isoform b and creates a reading-frame shift in the isoform a, also creating a likely null allele (Fig. 2A). 
Both spop-1 mutations recapitulated the RNAi phenotypes observed for both age-dependent paralysis (Fig. 2E,F) and growth arrest (Fig. 2B). To determine if spop-1 also functions to regulate neuronal DPR toxicity, we utilized a newly developed line expressing (GR)50 in the motor neurons. Motor neuron (GR)50 caused a progressive, age-dependent loss in motility (Fig. S4). The spop1(gk630214) mutant suppressed this motor neuron (GR)50 phenotype (Fig. S4). Therefore, loss of spop-1 function suppresses C9orf72-associated DPR toxicity in multiple cellular contexts.

In mammals, SPOP functions as an adaptor protein that targets substrates for ubiquitination by cullin 3-type E3 ubiquitin ligases and subsequent proteasomal degradation (26). If spop-1 confers protection against DPR toxicity through its canonical function as an E3 ubiquitin ligase adaptor, then inhibition of the cullin 3 ubiquitin ligase should phenocopy spop-1 mutants and protect against DPR toxicity. Furthermore, this protective effective should exhibit a nonadditive gene interaction with spop-1 mutants. Since cul-3 mutants are not viable, we tested these predictions by performing cul-3(RNAi) in the wild type and spop-1(dr28) mutant background. cul-3(RNAi) in a wild type background provided significant protection against (PR)50 toxicity (Fig. 3A). While spop1(dr28) also suppressed (PR)50 toxicity, cul-3(RNAi) in the spop-1(dr28) mutant did not provide additional suppression (Fig. 3A). This is unlikely to be due to a floor effect since $g f p(R N A i)$ consistently suppresses (PR)50 toxicity to a greater extent than spop-1(dr28) (Fig. 3A). Together, these genetic data suggest that 
cul-3 functions in the same genetic pathway as spop-1 to mediate DPR toxicity in C. elegans.

\section{Cancer-causing missense mutations in SPOP protect against (PR)50 toxicity}

Genetic missense mutations in SPOP are a common cause of prostate cancer. The most common mutations, W131G and F133V, are loss-of-function. These mutations are in the SPOP MATH domain and interfere with the ability of SPOP to interact with its substrates. If the role of SPOP in DPR toxicity is to target substrates for cul-3-dependent ubiquitination and degradation, then introduction of these mutations into $C$. elegans spop- 1 is predicted to phenocopy the spop-1 null alleles and confer protection against (PR)50 toxicity. We used CRISPR/Cas9 to introduce each mutation into the endogenous spop-1 gene (W195G and F197V in C. elegans) in the (PR)50 background. We also knocked these mutations into a spop-1-gfp allele tagged at the endogenous locus with CRISPR/Cas9. Like the spop-1 null alleles, both missense mutations conferred protection against (PR)50 toxicity (Fig. 3B). While spop-1W195G_-gfp exhibited normal abundance and nuclear localization, spop-1F197V-gfp showed a substantial decrease in the SPOP-1-GFP protein levels, suggesting the F197V mutation reduces SPOP-1 protein levels (Fig. 3C). Loss of function spop-1 alleles did not alter the levels or localization of either the (PR)50 or (GR)50 proteins, suggesting spop-1 functions downstream of DPR nuclear localization to mediate toxicity (Fig. 
3D). Together, these genetic data are consistent with the hypothesis that spop-1 mediates DPR toxicity via cul-3-dependent ubiquitination and degradation of one or more spop-1 substrates.

\section{SPOP is required for DPR toxicity in mammalian neurons}

SPOP is highly conserved from C. elegans to humans (Fig. 1), but is absent from yeast. To determine if the role of SPOP in DPR toxicity is evolutionarily conserved, we utilized a rat spinal cord neuron culture system model of DPR toxicity. Embryonic rat spinal cord motor neurons were infected with recombinant Herpes Simplex Virus (HSV) engineered to express miRNAs (miRs) targeting rat SPOP. Two independently derived SPOP miRs led to a $\sim 90 \%$ reduction in SPOP protein levels in pure neuronal cultures (Fig. 4A). After growing neurons on an astrocyte feeder layer for two weeks, we then co-infected our spinal cord neuronal cultures with two separate HSVs expressing a codonoptimized DPRs (GA50, (GR)50, or (PR)50) and the SPOP miR. In prior work, we show that both viruses express their cargo in $>95 \%$ of neurons in a non-toxic manner $(29,30)$. We found that expression of known toxic DPRs GA50, (GR)50, and (PR)50 caused significant neuronal toxicity and reduced the number of viable primary motor neurons $\sim 50 \%$ (Fig. $4 \mathrm{~B}$ ). Expression of a scrambled control sequence miR was neither toxic nor protected against the toxicity of (PR)50 (Fig. 4B). However, expression of the SPOP miR provided highly significant neuroprotection against the toxicity of all DPRs. Interestingly, while the SPOP 
miR robustly inhibited SPOP protein expression, the levels of several known SPOP ubiquitination substrates identified in cancer cells (e.g., PTEN, DUSP7, and G3BP1) were not altered by either (PR)50, (GR)50, or SPOP knockdown (Fig. 4C). Since these SPOP substrates have been largely characterized in renal, prostate, and endometrial cancer cell lines, these data present the possibility that neuronal SPOP functions may include the degradation of novel neuronal substrates. Finally, as we saw in C. elegans spop-1 mutants, SPOP knockdown in mammalian neurons did not alter the abundance of any of the toxic DPRs (either nominal monomers or higher molecular weight species; Fig. 4D). These data suggest that SPOP is an evolutionarily conserved factor broadly required for DPR toxicity from $C$. elegans to mammalian neurons and that it functions downstream of DPRs to engage mechanisms leading to cellular toxicity.

SPOP is strongly upregulated in most clear cell renal carcinomas and genetic inhibition of SPOP in these cancer cells attenuates their growth (31). As a result, Guo et al performed a small molecule screen for SPOP inhibitors (32). One inhibitor, Compound 6b, blocks SPOP-substrate interactions with low micromolar affinity. We asked whether $6 \mathrm{~b}$ was neuroprotective in our tissue culture model. Application of $6 \mathrm{~b}$ had no effect on motor neuron survival in naive cultures (not shown). However, 6b exhibited a statistically significant blunting of DPR toxicity in cultures that express (GR)50, GA50 or the exogenously provided PR20 peptide (Fig. S5) $(14,19,33)$. These observations suggest that the 
SPOP-CUL3 pathway we have defined may be amenable to small molecule modulation in mammals.

\section{The bromodomain containing protein BET-1 is required for spop-1 mutant suppression of DPR toxicity}

Our data suggest a model in which a conserved function of SPOP leads to the ubiquitin-dependent degradation of one or more substrates when toxic DPRs are present. In an SPOP mutant, this substrate(s) is no longer ubiquitinated and degraded, leading to increased protein levels and protection against DPR toxicity. A prediction of this model is that reducing the abundance of this substrate should reverse the protective effect of spop-1 mutants against DPRs. To identify such substrates, we performed a spop-1 mutant suppressor screen. In mammalian cancer cells, spop-1 targets $>30$ proteins for degradation (26). Eleven of these substrates have homologs in $C$. elegans. We performed feeding-based RNAi and paralysis assays for 9 of these candidate SPOP substrates in both the WT and spop-1(dr28) mutant. Only a single gene knockdown against the BRD2/3/4 homolog bet-1 suppressed spop-1(dr28) protection against DPR toxicity (Fig. 5). In wild type animals, bet-1(RNAi) did not further enhance DPR toxicity (Fig. 5A). bet-1(RNAi) also suppressed the paralysis phenotypes of the spop-1 LOF allele gk630214 (Fig. 5B) and the spop1 W195G missense allele (Fig. 5C). This indicates that the bet-1(RNAi) suppression of spop-1 is not allele specific. Rather, loss of bet-1 suppresses the 
phenotypic consequences associated with loss of spop-1. Taken together, these genetic data are consistent with a model in spop-1 mediates the degradation of bet-1 and that stabilization of bet-1 protects against DPR toxicity (Fig. 5D).

\section{Discussion}

Aggregated, misfolded, and otherwise insoluble proteins, including DPRs, accumulate in the brains of individuals who bear the HRE in the C9ORF72 locus and these species are widely believed to be key contributors to disease pathophysiology. One potential driver of this process is direct inhibition of the proteasome by DPRs. Cryo-electron tomographic imaging of cells expressing GA DPRs show that twisted GA filaments decorated with proteasomes, which appear arrested in a non-functional state (13). Similarly, PR DPRs directly bind to purified proteasomes and inhibit degradation of ubiquitinated substrates (14). Here we define components of a novel pathway that also ties the ubiquitinproteasomal degradation pathway to DPR toxicity. In our unbiased RNAi screen, we discovered that the E3 ubiquitin ligase adaptor SPOP and its associated E3 ligase, cullin 3, are required for DPR toxicity. The beneficial effects of ablation of SPOP, as well as introduction of mutations that inactivate its ability to present clients to the E3 ligase, provide strong evidence that the canonical ubiquitination function of SPOP is indispensable for DPR toxicity.

Previously, we have shown that the toxicity of PR and GR DPRs are dependent upon nuclear localization (20). This aligns well with the fact that 
SPOP is also a predominantly nuclear protein and suggests that SPOPdependent degradation of a nuclear protein(s) renders cells vulnerable to DPR toxicity. SPOP was originally identified in 1997 as a POZ domain containing protein that localizes to subnuclear speckles (34). Subsequent studies revealed that SPOP speckles have biophysical characteristics associated with proteins that undergo liquid-liquid phase separation (LLPS) (35). Moreover, SPOP nuclear speckles are highly dynamic and are enriched with known SPOP ubiquitination substrates (36). Given that PR and GR dipeptides can both undergo LLPS themselves and interfere with LLPS associated with other proteins $(17,18,37)$, our discovery that SPOP is required for DPR toxicity suggests the possibility that alterations in the LLPS properties of SPOP speckles by (PR)50 could be the basis for DPR toxicity. These alterations could either occur through direct or indirect SPOP-DPR interactions. For example, DPRs could directly interact with SPOP and block its ability to co-localize with and degrade specific ubiquitination targets. An alternative hypothesis is that DPRs could drive SPOP to inappropriately interact with and degrade targets that would not normally be degraded. Our data demonstrating that inhibition of the SPOP substrate bet-1 suppresses the protective effect of SPOP inhibition on DPR toxicity supports the latter hypothesis. Additional studies examining SPOP-DPR interactions, as well as the roles of other SPOP substrates in mammalian cells, are needed to further differentiate between these possibilities.

Our studies are the first to link SPOP to a neurodegenerative disease. However, SPOP has well characterized links to non-neuronal diseases, such as 
prostate, endometrial, and renal cancer $(31,38,39)$. In prostate cancer, recurrent somatic missense mutations such as W131G and F133V occur in $\sim 10 \%$ of metastatic patients (40). These mutations lead to SPOP loss of function and stabilization of multiple SPOP substrates $(41,42)$. Many additional missense mutations are associated with SPOP-dependent cancer, including several gain-of-function mutations associated with endometrial cancer that enhance the degradation of SPOP substrates (43). A prediction of our findings is that such gain-of-function alleles may enhance DPR toxicity. The highly conserved nature of the SPOP protein from $C$. elegans to humans, combined with efficient CRISPR engineering in worms and highly penetrant DPR phenotypic assays, provide a unique opportunity to define SPOP structurefunction relationships in SPOP-dependent DPR toxicity in ways that are not possible in other systems.

SPOP targets many proteins for degradation (44). In SPOP prostate cancer mutants, the ability to target and degrade these substrates is lost, which leads to their upregulation (36). Upregulation of several such SPOP substrates are associated with different aspects of the cancer phenotype $(43,45,46)$. One important set of substrates are the Bromodomain and Extra-Terminal motif (BET) containing proteins BRD2, BRD3, and BRD4. BET proteins are transcriptional co-activators that interact with acetylated histones and transcription factors to control gene expression (47). Our studies here show that BET proteins are also likely to play a role in SPOP-dependent DPR toxicity. We discovered that the protective effect of spop-1 mutants on DPR toxicity was suppressed by reducing 
the abundance of the $C$. elegans protein bet-1. Our findings suggest a model in which DPRs lead to the inappropriate spop-1-dependent degradation of bet-1, and possibly other substrates. Loss of spop-1 leads to BET-1 upregulation, which protects against DPR toxicity via alterations in gene expression. Inhibition of bet-1 in the spop-1 mutant suppresses these compensatory transcriptional responses and restores DPR toxicity. While our genetic studies are consistent with this model, additional cell biological and biochemical tests are needed to more rigorously test its predictions. Interestingly, in a recent small molecule screen, two BET inhibitors were identified as suppressors of exogenous PR20 toxicity (48). These observations are nominally the opposite effect our model would predict. The disparate results may be due to the differences in discovery platforms, read-out measures, redundant effects on multiple BRD domain proteins, and/or off-target drug effects. In this respect, genetic studies may be a more precise tool for investigating the role of BET proteins in DPR toxicity.

The primary goal of research into disease mechanism is to find a druggable target. In this regard, SPOP is an attractive candidate. The SPOP inhibitor Compound 6b stops the growth of SPOP overexpressing ccRCC cells in culture and attenuates tumor growth in a mouse model. A recent study identified $6 \mathrm{~b}$ derivatives that are even more potent (49), suggesting that the SPOP pathway is highly suitable for small molecule intervention. Given that genetic inhibition of the cancer-relevant functions of SPOP can also suppress DPR toxicity, we predict that $6 \mathrm{~b}$ and its derivatives could also be a new and potentially viable therapeutic option for opposing DPR toxicity. Preclinical studies show that 
$6 \mathrm{~b}$ is well tolerated and has no major side effects (32), although the blood-brain barrier permeability of this compound is not known. Future studies investigating $6 \mathrm{~b}$ or other SPOP inhibitors could provide a novel entry point for new ALS/FTD therapies.

\section{Conclusion}

In conclusion, our unbiased genetic screening approach in $C$. elegans discovered that the SPOP ubiquitin ligase pathway is essential for C9 DPR toxicity. Given that SPOP is a nuclear protein and DPR nuclear localization is required for toxicity, our findings provide additional evidence that the mechanism of DPR toxicity originates in the nucleus. Drugs targeting both SPOP and its relevant targets, such as BET domain proteins, are useful tools for treating cancer and could be equally useful as C9 disease therapeutics. 


\section{Materials and methods}

\section{C. elegans strains, culture, and genome-wide RNAi screen}

See Table 2 for a list of the strains utilized in this study. Unless otherwise indicated, animals were cultured at $20^{\circ} \mathrm{C}$. The genome wide RNAi screen utilized the Ahringer feeding library (Source Bioscience, Nottingham, U.K.) and was performed as previously described $(50,51)$. Briefly, 96 well plates containing $\sim 100 \mu \mathrm{l}$ of LB $+25 \mu \mathrm{g} / \mathrm{ml}$ carbenicillin liquid media were seeded with individual bacterial clones using a 96 well pin tool and grown for $18-24$ hours at $37^{\circ} \mathrm{C} .20 \mu \mathrm{l}$ of this overnight culture was seeded into a single well of a 24 well plate containing NGM agar $+25 \mu \mathrm{g} / \mathrm{ml}$ carbenicillin + 1mM IPTG (Fisher Scientific) and incubated overnight at room temperature $\left(\sim 20^{\circ} \mathrm{C}\right)$. Prior to seeding on RNAi screening plates, the drls34 strain is fed $g f p(R N A i)$ bacteria to suppress the expression of (PR)50-GFP, which allows drls34 animals to grow and reproduce. When fed empty vector(RNAi) or standard OP50, the drls34 strain produces progeny that are growth arrested and paralyzed. This phenotype is $100 \%$ penetrant. Therefore, $g f p(R N A i)$ serves as a positive control for the (PR)50-GFP suppression screen and EV(RNAi) serves as a negative control. 30 synchronized L1 stage drls34 worms were seeded into each well and incubated at $20^{\circ} \mathrm{C}$. After 7 days, each well was manually screened for RNAi clones that allowed further growth and/or restored mobility compared to the $E V(R N A i)$ negative control. 'Hits' from this primary screened were re-screened six times and clones that were identified as 'hits' in 4/6 rescreens were considered validated hits. During the screening process, the experimenters were blinded to the identity of the RNAi clone. RNAi clones from 
validated hits were sequenced to determine the identity of the gene targeted. These sequence-validated strains were frozen as glycerol stocks and used for all subsequent RNAi-based experiments.

\section{Molecular Biology and CRISPR methods}

All procedures involving recombinant or synthetic nucleic acid molecules and materials were approved by the University of Pittsburgh Institutional Biosafety Committee. The drls34 ((PR)50) and drls28 ((GR)50) transgenes were previously described (20). CRISPR/Cas9 engineering was performed according to the method of Ghanta et al (52). For point mutations and deletion edits, worms were injected with a mixture of Cas9 (250ng/ $\mu$ l (IDT)), tracrRNA (100 $\mathrm{ng} / \mu \mathrm{l}$ (IDT)), guide

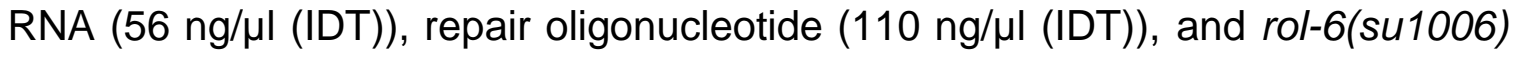
marker plasmid (40 ng/ $\mu \mathrm{l})$. The Cas9, tracrRNA, and guide RNA was incubated at $37^{\circ} \mathrm{C}$ for 10 minutes prior to the addition of the repair oligo and rol-6 plasmid. For generation of the spop-1-GFP CRISPR allele, a gfp PCR product was PCR amplified from pPD95.75 (Addgene) using primers containing 35bp of overlap with the spop-1 gene immediately upstream of the predicted nuclear localization sequence. Guide RNAs, repair oligonucleotides, PCR primers, and genotyping primers used in this study are listed in Table 3.

\section{Microscopy}

Low magnification images were captured on a MZ16FA fluorescence stereo microscope equipped with a DFC345 FX camera and AF imaging software (Leica 
Microsystems). For high magnification images, day 1 adult hermaphrodites were anesthetized in $10 \mathrm{mM}$ levamisole and imaged within a silicon grease enclosed slide chamber. Animals were imaged on a wide-field DMI4000B inverted microscope with a $63 X$ oil immersion lens. Images were captured with a DFC 340 FX digital camera using AF imaging software (Leica Microsystems). All images were captured with identical exposure times and image brightness/contrast/intensity was adjusted for all images within a panel simultaneously within CorelDraw X7.

\section{Paralysis and thrashing assays}

DPR paralysis assays were performed as previously described $(20,53)$. For the thrashing assay quantification of the integrated (GR)50 motor neuron line, 10 L4 animals were picked onto 3cm NGM plates spotted with OP50 (5 plates per genotype). At each time point, animals were picked into a dish containing $\sim 1 \mathrm{ml}$ of $\mathrm{M9}$ solution. After a 5 ' acclimation to liquid, the number of thrashes in 30 seconds was quantified. Each time the nose of the animals crossed the body midline was considered a thrash. 10 worms per genotype were measured at each time point. At the completion of the assay, the thrashing rates for each genotype at each time point were normalized to the 'Day 0' thrashing rate for that genotype in order to control for any baseline differences in thrashing. The experimenter counting the thrash rates was blinded to the genotype. Each experiment was performed 2-3 times and the results from a single representative experiment are shown. 


\section{Mammalian neuronal cultures}

Primary neurons cultures were established according to previously described methods $(14,29,30)$. Briefly, cortical astrocytes from newborn rat pups were plated on German Glass, acid washed glass coverslips (18 millimeter round), grown in Minimal Essential Media MEM) $+10 \%$ fetal bovine serum and 10\% horse serum (HS) and when they achieved 60\% confluency, dissociated embryonic (E) day 15 rat spinal cord neurons were added. The media was changed to astrocyteconditioned MEM + 10\% HS + glial derived neurotrophic factor, ciliary neurotrophic factor and cardiotropin-1 (all at $2 \mathrm{nM})$. AraC (10uM) was then added to arrest astrocyte proliferation and washed out with subsequent bi-weekly media supplementation. After 14 days in vitro (DIV), recombinant HSV were added and cultures were fixed five days later. The SPOP miR clone was designed using the BLOCK-IT miR RNAi design tool (SPOP accession number NM_001100496.1; ThermoFisher Scientific). Sense and antisense oligos for three distinct rat SPOP miRs beginning at SPOP cDNA basepair 212, 239, and 336 were synthesized and cloned into the miR expression vector p1006 for packaging into HSV. Western blot validation experiments in HEK cells using overexpressed SPOP showed that the 336-miR was slightly less effective than the $212-$ or $239-\mathrm{miR}$. Therefore, only the 212-miR and 239-miRs were utilized. Viruses were generated by the Gene Delivery Technology Core at Massachusetts General Hospital.

For pure neuron biochemical studies, E17 rat cortex was dissociated and plated on plastic petri dishes and maintained in astrocyte-conditioned, Neurobasal + B27 supplement. After 14 DIV recombinant HSV was added and lysates 
prepared for western blotting. Antibodies used were: anti-GA (1:1000; Millipore Sigma), anti-GR (kind gift of Q. Zhu, Cleveland lab), anti-PR (kind gift of Y. Zhang, Petrucelli lab), anti-SPOP (1:500; Proteintech), anti-tubulin (1:5000; BioLegend), anti-PTEN (1:1000; Cell Signaling Technology), anti-DUSP7 (1:1000; Abcepta), and anti-G3BP1 (1:1000; Cell Signaling Technology).

\section{Motor neuron toxicity assay}

Methodological details of the in vitro mammalian motor neuron toxicity assay have been reported previously $(29,30,54,55)$. In brief, coverslips containing paraformaldehyde fixed astrocyte/spinal cord co-cultures were stained with SMI32 antibody - when restricted to neurons with a cell body diameter of 20 microns or greater, a validated marker of alpha motor neurons in this culture system (56). The number of motor neurons in three randomly selected $20 x$ lower power field was determined and averaged. The average value of four coverslips was determined (experimental replicates). Determination of motor neuron number was obtained with the counter blinded to the experimental manipulation. The results from 3+ independent experiments are presented.

\section{Statistical Analysis}

Paralysis assays were analyzed using the Kaplan-Meier log-rank function (OASIS $(57,58))$. Comparisons of means were analyzed with either a two-tailed Students t-test (2 groups) or 1- or 2-way ANOVA (3 or more groups) using the 

available under aCC-BY-NC-ND 4.0 International license.

Tukey or Dunn's post-test analysis as indicated in GraphPad Prism 7 (GraphPad

Software, Inc., La Jolla, CA). p-values of $<0.05$ were considered significant. 


\section{Acknowledgements}

This work was supported by grants from the NIH (NS094921 and NS096319 to T.L., NS05225 and NS087077 to R.G.K). Research in the Kalb lab is support by the Les Turner ALS Center. We thank the lab of Dr. Caiguang Yang (Shaghai Institute of Materia Medica, Chinese Academy of Sciences) for generously providing Compound $6 \mathrm{~b}$, the Petrucelli lab (Mayo Clinic) for generously providing the anti-GA antibody, and the Cleveland lab (UCSD) for generously providing the anti-GR antibody. Some strains were provided by the CGC, which is funded by the NIH Office of Research Infrastructure Programs (P40 OD010440). 


\section{Figure legends}

Figure 1. A genome-wide RNAi screen for suppressors of (PR)50 toxicity in C. elegans. A) RNAi screening strategy. $\sim 30$ hypochlorite isolated eggs from the drls34 (myo-3p::(PR)50-GFP; myo-3p::mCherry) strain are seeded onto 24 well RNAi plates. After 7 days, each well was visually examined and scored relative to the empty vector(RNAi) control, which exhibits no growth and strong paralysis. Suppressors are identified as wells in which worms continue to grow and/or regain motility ( $g f p(R N A i)$ shown as an example). B) COPAS quantification of worm length (time-of-flight(TOF)) in RNAi suppressors of (PR)50. Worms with a TOF $>200$ were placed in the 'Growth' group while those with TOF $<200$ were placed in the 'No growth' group. Data are presented as the percentage of the total population in either the 'Growth' or 'No growth' groups. The size of each population $(\mathrm{N})$ is indicated at the top of each bar. ${ }^{* * * *}-\mathrm{p}<0.0001$, Fisher's exact test versus empty vector(RNAi). N.D. - not determined due to completely penetrant lethal phenotype in this replicate. C) Age-dependent paralysis assay was performed with homozygous viable mutations in genes identified in the RNAi screen. $N>50$ for each genotype. ${ }^{* * * *}-p<0.0001$, Log-Rank test with Bonferroni correction. D) Localization of (PR)50-GFP in either the WT or indicated homozygous mutant background. Arrows point to muscle nucleus. In WT, (PR)50-GFP is primarily localized to nuclei but in $n 4256$ and ok798, (PR)50-GFP is largely excluded from the nucleus and accumulates in non-nuclear compartments. Scale bar $=10$ microns. 
Figure 2. Loss of function mutations in the E3 ubiquitin ligase adaptor spop1 suppress (PR)50 and (GR)50 toxicity in C. elegans. A) Domain structure of the SPOP protein. The percent identity between the human and C. elegans domains are shown. The dr28 allele is a CRISPR-induced allele that deletes $82 \mathrm{bp}$ within the second exon of spop-1. This shifts the reading frame of isoform 1 to encounter a premature stop codon and deletes that start ATG for isoform 2. Therefore, $d r 28$ is a likely spop-1 null allele. The gk630214 allele is a spop-1 nonsense mutation isolated from the Million Mutation project. The gfp insertion site used to make the SPOP-1-GFP CRISPR allele is shown. B) spop1(gk630214) and ufd-2(tm1380) suppress the developmental arrest phenotype of (PR)50-GFP. C,D) spop-1(RNAi) suppresses the age-dependent paralysis phenotype of (PR)50-GFP (C) an (GR)50-GFP (D). E,F) spop-1 alleles gk630214 and dr28 suppress (PR)50-GFP (E) and (GR)50-GFP (F). For all paralysis assays, $N=50-100$ animals for each genotype, ${ }^{*}-p<0.05,{ }^{* * *}-p<0.001$, Log-rank test with Bonferroni correction for multiple testing.

Figure 3. Inhibition of a spop-1-cul-3 pathway protects against DPR toxicity without altering DPR levels or localization. A) (PR)50 paralysis assay in the indicated genetic backgrounds. $N=100$ animals per genotype. ${ }^{*}-p<0.05,{ }^{* *}$ $p<0.01,{ }^{* * *}-p<0.001,1$ way ANOVA with Tukey post-hoc comparisons. B) (PR)50 paralysis assay in the indicated spop-1 genotypes. Mutations were introduced into 
the endogenous C. elegans spop-1 gene via CRISPR/Cas9. $N=100$ animals per genotype. $\quad{ }^{*}-p<0.05,{ }^{* * *}-p<0.001,1$ way ANOVA with Tukey post-hoc comparisons. C) Localization of a CRISPR-engineered SPOP-1-GFP fusion protein containing the indicated point mutations. Germline nuclei are shown because their high density facilitates observation of multiple instances of SPOP-1GFP speckling. Similar speckling behavior is observed in somatic cells. Speckling was not observed in SPOP-1-GFPW195G. SPOP-1-GFPF197V expression was at the limit of detection and speckling could not be evaluated. Scale bar = 10 microns. Boxed regions are enlarged, deconvolved images to illustrate SPOP-GFP speckling. Scale bar in enlarged image $=5$ microns. D) (PR)50-GFP and (GR)50GFP expression in wild type or spop-1(gk630214). Images are exposure matched. Arrows point to muscle nuclei with nucleolar DPR enrichment, as was previously described for (PR)50-GFP and (GR)50-GFP localization in C. elegans (20). Scale bar $=10$ microns

Figure 4. SPOP knockdown protects against DPR toxicity in mammalian primary neurons without reducing the abundance of known SPOP ubiquitination targets or DPRs themselves. A) Cortical neuron cultures were infected with Herpes Simplex Virus (HSV) engineered to express LacZ, a scrambled sequence microRNA (miR) or two different miRs targeting SPOP. Endogenous neuronal SPOP levels are reduced by targeted miRNAs. B) Days in vitro14 (DIV14) spinal cord neuron cultures were co-infected with HSV engineered to express the indicated DPR and either the scrambled sequence miR (control - 
"cont") or miR targeting SPOP. Control cultures were infected with HSV-LacZ. Five days later, cultures were processed for immunocytochemistry and motor neuron counts were obtained. By ANOVA, DPRs lead to a statistically significant reduction in motor neuron number (compared with LacZ expressing cultures) and knockdown of SPOP leads to a statistically significant protection against SPR toxicity (e.g., $\mathrm{GA}(50)$ group, $F_{(3,48)}=84.8$, p < 0.001; $\mathrm{GR}(50)$ group, $F_{(3,47)}=240.6$, $\mathrm{p}<0.0001 ; \mathrm{PR}(50)$ group, $\left.F_{(3,48)}=119.0, \mathrm{P}<0.0001\right)$. C) Cortical neuron cultures were infected with HSV engineered to express LacZ or the GA(50), (PR)50 or (GR)50 DPRs. Simultaneously, cultures were infected with either the HSV engineered to express the scrambled sequence miR or SPOP miR. Two days later, before any neuron loss is detected, lysates were probed for known SPOP targets. No statistically significant group differences were detected in scan band intensities by ANOVA (not shown). D) Lysates from experiments described in C) were probed for GA, GR or PR and imaged with short or long exposures (to see less abundant higher molecular weight species). Putative monomers are noted with ">". No statistically significant group differences were detected in scan band intensities by ANOVA (not shown).

Figure 5. spop-1 mutant protection against DPR toxicity requires the bromodomain protein bet-1. A) Paralysis assay with the indicated genotypes. bet-1 null alleles are homozygous sterile (59). Therefore, bet-1(RNAi) was utilized. All genotypes include the drls34 (myo-3p::3XFLAG-(PR)50-GFP) transgene. $\mathrm{N}=100$ animals per genotype. ${ }^{* * *}-p<0.001$, n.s. $-p>0.05$, Log-Rank test with 
Bonferroni correction for multiple comparisons. B) Paralysis assay in the indicated spop-1 allele $+/-$ bet-1(RNAi). $\mathrm{N}=100$ animals per genotype. ${ }^{* * *}-\mathrm{p}<0.001$, LogRank test with Bonferroni correction for multiple comparisons C) Paralysis assay in the indicated spop-1 allele +/- bet-1(RNAi). $\mathrm{N}=100$ animals per genotype. ${ }^{* * *}$ $p<0.001$, Log-Rank test with Bonferroni correction for multiple comparisons $D$ ) Model for the function of SPOP and BRD proteins in C9 DPR toxicity.

Figure S1. Quantification of suppression of (PR)50-induced growth arrest by hits from the genome-wide RNAi screen. drls34 eggs were placed on either (empty vector) RNAi or the indicated gene-specific RNAi bacteria. After 7 days at $20^{\circ} \mathrm{C}$, animals were washed off the plate and worm length was quantified as the event time-of-flight using a COPAS Biosort. Data shown are mean +/- S.D. The $\mathrm{N}$ for each sample is shown on each bar. $\mathrm{P}$-values versus the empty vector control were calculated using a non-parametric one-way ANOVA with Dunn's multiple comparison post-hoc testing.

Figure S2. Effect of (PR)50 RNAi screen hits on (GR)50 age-dependent paralysis phenotype. Paralysis assays were performed as previously described (60). $N=50-80$ animals per assay. $E V(R N A i)$ control in black and gene specific RNAi in red. Each candidate gene was scored in two separate assays and the number of times the Log-rank Bonferroni-adjusted $p$-value was $<0.05$ is indicated. 
Figure S3. Effect of RNAi screen hits on transgene expression. drls33 eggs were placed on either empty vector(RNAi) or the indicated gene-specific RNAi bacteria. After 7 days at $20^{\circ} \mathrm{C}$, animals were washed off the plate and normalized GFP (GFP/TOF; top) and normalized RFP (RFP/TOF; bottom) from adult animals with TOF $>400$ were quantified with a COPAS Biosort. Data shown are mean +/S.D. ${ }^{*}-p<0.05,{ }^{* * * *}-p<0.001$, non-parametric One-way ANOVA with Dunn's multiple comparisons test. The $\mathrm{N}$ for each sample is indicated within each bar.

Figure S4. spop-1 functions in motor neurons to protect against motor neuron expressed (GR)50-GFP. WT or drls49 (unc-47p::3xFLAG-(GR)50-GFP) animals of the indicated genotype were grown at $25^{\circ} \mathrm{C}$ and L4 stage animals (Day 0) were picked to new plates ( 4 sets of plates with 10 animals each per genotype). At each timepoint, the number of thrashes were quantified as previously described (60). The experimenter was blinded to genotype. Data within genotypes were normalized to the thrashing rate at Day 0 . Data shown are the mean \pm S.D., $N=10$. ${ }^{* * *}-p<0.001$.

Figure S5. The SPOP inhibitor, compound 6B protects against DPR toxicity.

DIV14 spinal cord neuron cultures were infected with HSV engineered to express the $\mathrm{GA}(50), \mathrm{GR}(50)$ or LacZ or exposed to bath applied synthetic PR20 and were treated with either $6 \mathrm{~B}(10 \mathrm{uM})$ or vehicle once. Five days later, cultures were processed for immunocytochemistry and motor neuron counts were obtained. By 
one-way ANOVA, DPRs lead to a statistically significant reduction in motor neuron number (compared with LacZ expressing cultures) and application of $6 \mathrm{~B}$ leads to a statistically significant protection against DPR toxicity (e.g., GA(50) group, $F(2,69)$ $=49.85, \mathrm{p}<0.0001 ; \mathrm{GR}(50)$ group, $\mathrm{F}(2,51)=114.0, \mathrm{p}<0.0001 ; \mathrm{PR} 20$ group, $\mathrm{F}(2,129)$ $=79.32, \mathrm{p}<0.0001$. 
Table 1. RNAi gene knockdowns that suppress myo-3p::(PR)50-GFP toxicity

\begin{tabular}{|c|c|c|c|c|c|c|c|}
\hline $\begin{array}{l}\text { C. } \\
\text { elegans }\end{array}$ & Yeast & Fly & Human & Description & $\begin{array}{l}\text { Predicted or } \\
\text { demonstrated } \\
\text { NLS }\end{array}$ & $\begin{array}{l}\text { Suppresses } \\
\text { (GR)50? }\end{array}$ & Lethal/Sterile \\
\hline \multicolumn{8}{|c|}{ Nuclear transport / RNA binding } \\
\hline$r s p-6$ & MRD1 & $\mathrm{X} 16^{2}$ & SRSF $^{1}$ & $\begin{array}{l}\text { RNA binding } \\
\text { protein }\end{array}$ & Yes & Yes & Yes \\
\hline$z t f-4$ & NGR1 & CG42458 ${ }^{2}$ & $\mathrm{NCOA} / \mathrm{hnRNPC}^{1}$ & $\begin{array}{l}\text { contains RRM } \\
\text { domain, similar to } \\
\text { hnRNPC }\end{array}$ & Yes & Yes & Yes \\
\hline$g y f-1$ & SYH1 & Gigyf & GIGYF $^{1}$ & $\begin{array}{l}\text { yeast homolog } \\
\text { SYH1 binds } \\
\text { proline-rich } \\
\text { sequences and } \\
\text { influences } \\
\text { nuclear pore } \\
\text { distribution }\end{array}$ & Yes & Yes & No \\
\hline ima-3 & KAP122 ${ }^{4}$ & Kap- $\alpha 3^{2,3}$ & KPNA3 $^{1}$ & $\begin{array}{l}1 \text { of } 3 \text { nuclear } \\
\text { importin alpha } \\
\text { subunits }\end{array}$ & No & No & Yes \\
\hline ergo-1 & None & AG01T1 & AGO3 & $\begin{array}{l}\text { Production of } \\
\text { endo-siRNAs }\end{array}$ & Yes & Yes & No \\
\hline \multicolumn{8}{|c|}{ Ubiquitin-mediated protein degradation } \\
\hline spop-1 & None & roadkill & SPOP & $\begin{array}{l}\text { Speckle-type } \\
\text { POZ protein; no } \\
\text { yeast homolog }\end{array}$ & Yes & Yes & No \\
\hline ufd-2 & Ufd2 & $\begin{array}{l}\text { UBE4B/ } \\
\text { CG9934 }\end{array}$ & UBE4a & $\begin{array}{l}\text { E3 and/or E4 } \\
\text { ubiquitin ligase }\end{array}$ & Yes & Yes & No \\
\hline rpn-12 & RPN12 & RPN12 & PSMD8 $^{5}$ & $\begin{array}{l}\text { nonATPase } \\
\text { regulatory subunit } \\
\text { of the } 26 S \\
\text { proteasome }\end{array}$ & No & Yes & No \\
\hline$r p n-9$ & RPN9 & RPN9 & PSMD13 & $\begin{array}{l}\text { nonATPase } \\
\text { regulatory subunit } \\
\text { of the } 26 \mathrm{~S} \\
\text { proteasome }\end{array}$ & No & Yes & Yes \\
\hline \multicolumn{8}{|c|}{ Chromatin regulation } \\
\hline met-2 & MET2 & eggless & SETDB1 & $\begin{array}{l}\text { Histone } \\
\text { methyltransferase }\end{array}$ & Yes & Yes & Yes \\
\hline utx-1 & CYC8 & Utx & UTX/UTY $^{6}$ & $\begin{array}{l}\text { Histone } \\
\text { demethylase }\end{array}$ & Yes & Yes & Yes \\
\hline pis-1 & None & PTIP & PAXIP1 & $\begin{array}{l}\text { Pax transcription } \\
\text { factor interacting } \\
\text { protein }\end{array}$ & Yes & No & Yes \\
\hline
\end{tabular}

1 - Interacts with both PR and GR in human cells (18)

2 - Enhanced the toxicity of (GR)50 in Drosophila model (18)

3 - Enhanced the toxicity of (PR)25 in Drosophila model (21)

4 - Suppresses the toxicity of (PR) 50 when overexpressed in yeast (9)

5 - Gene is enriched or depleted in (PR)20 treated K562 cell CRISPR screen

(19)

6 - Gene is enriched or depleted in (GR)20 treated K562 cell CRISPR screen

(19) 
Table 2. Strains used in this study

\begin{tabular}{|c|c|c|}
\hline Strain name & Genotype & Reference \\
\hline OG736 & $\begin{array}{l}\text { drls28 [myo- } \\
\text { 3p::3XFLAG-(GR)50-gfp; } \\
\text { myo-3p:dsRed2] }\end{array}$ & (20) \\
\hline OG755 & $\begin{array}{l}\text { drls34 [myo- } \\
\text { 3p::3XFLAG-(PR)50-gfp; } \\
\text { myo-3p:dsRed2] }\end{array}$ & (20) \\
\hline OG919 & met-2(n4256); drls34 & This study \\
\hline OG922 & rsp-6(ok798); drls34 & This study \\
\hline VC40422 & spop-1(gk630214) & (61) \\
\hline OG1053 & spop-1(gk630214) drls34 & This study \\
\hline OG1054 & $\begin{array}{l}\text { spop-1(gk630214); } \\
\text { drls28 }\end{array}$ & This study \\
\hline OG1055 & $\begin{array}{l}\text { spop-1(dr26(CRISPR } \\
\text { inserted gfp)) }\end{array}$ & This study \\
\hline OG1057 & spop-1(dr28); drls34 & This study \\
\hline OG1153 & $\begin{array}{l}\text { drls } 49 \text { [unc- } \\
47 p:: 3 X F L A G-(G R) 50- \\
\text { gfp; unc-47p:::gfp; myo- } \\
\text { 3p::mCherry] }\end{array}$ & This study \\
\hline OG1158 & $\begin{array}{l}\text { spop-1(dr95(W195G)); } \\
\text { drls34 }\end{array}$ & This study \\
\hline OG1159 & $\begin{array}{l}\text { spop-1(dr26 } \\
d r 96(W 195 G))\end{array}$ & This study \\
\hline OG1168 & $\begin{array}{l}\text { spop-1(dr100(F197V); } \\
\text { drls34 }\end{array}$ & This study \\
\hline OG1169 & $\begin{array}{l}\text { spop-1(dr26 } \\
\text { dr101(F197V)) }\end{array}$ & This study \\
\hline OG1171 & $\begin{array}{l}\text { spop-1(gk630214); } \\
\text { drls49 }\end{array}$ & This study \\
\hline
\end{tabular}


Table 3. Oligonucleotides used in this study

\begin{tabular}{|c|c|c|}
\hline Primer & Sequence & Description \\
\hline TL118 & GAGTCAGTGAGCGAGGAAGC & $\begin{array}{l}\text { C. elegans feeding RNAi clone } \\
\text { sequencing primer }\end{array}$ \\
\hline OG1454 & TACCGTACTACACGCATCGCGAGC & rsp-6(ok798) genotyping \\
\hline OG1455 & CGAGAGCGGGAGCGACTCTTCGAG & rsp-6(ok798) genotyping \\
\hline OG1456 & CGCGTTTATTGAAATCATTTCGCG & rsp-6(ok798) genotyping \\
\hline OG1457 & ACGATCACGTTCTTGAGGAGATCG & rsp-6(ok798) genotyping \\
\hline OG1458 & GCAGGAAAGATGTATTCGACAAATATGC & rsp-6(ok798) genotyping \\
\hline OG1449 & GTGAATCACTTGAGGGACTTTCAC & met-2(n4256) genotyping \\
\hline OG1450 & GTCCAACCATAATAGCAATCTGAGC & met-2(n4256) genotyping \\
\hline OG1451 & GATGAAGACGTGCTAGTCGCTGC & met-2(n4256) genotyping \\
\hline OG1452 & CCTGGTTCACCAGGCATTGTCGGAG & met-2(n4256) genotyping \\
\hline OG1453 & CAGGTCTTCGAGTTGCCTGTCGCC & met-2(n4256) genotyping \\
\hline OG1534 & AGATCTCCAGTCGCCTTTTC & spop-1(dr28) guide RNA 1 \\
\hline OG1535 & CGGCATGGGTAACGATGAAG & spop-1(dr28) guide RNA 2 \\
\hline OG1537 & $\begin{array}{l}\text { AATTAACAAATAAATATATTTGAATTTAAGATAAATAAGTGTAAAT } \\
\text { TTAATTTACAAATACTGTAAACGA }\end{array}$ & spop-1(dr28) repair oligo \\
\hline OG1528 & AGCTCGTGGCAAGTGTGCATAC & spop-1(dr28) genotyping \\
\hline OG1529 & GTTTCCCGATCCACTCGAGACCTG & spop-1(dr28) genotyping \\
\hline OG1459 & GTGCCGAAAACAGTCTGGCAATGG & spop-1(gk630214) genotyping \\
\hline OG1460 & GAGATCCACTTCTTCCGGTTGCTG & spop-1(gk630214) genotyping \\
\hline OG1461 & CAGTCTGGCAATGGACAAAC & spop-1(gk630214) genotyping \\
\hline OG1462 & CCGGTTGCTGAAAATTGGTGTC & spop-1(gk630214) genotyping \\
\hline OG1536 & GGCTGTACAAGCACTACGGG & spop-1(dr26)-gfp gRNA \\
\hline OG1530 & $\begin{array}{l}\text { TTTAGAGCACTAGCAACTCAGCAAACTCCTCCCGTAATGAGTAA } \\
\text { AGGAGAAGAACTTTTC }\end{array}$ & $\begin{array}{l}\text { GFP PCR with spop- } 1 \text { overlaps, } \\
\text { CRISPR repair template }\end{array}$ \\
\hline OG1531 & $\begin{array}{l}\text { GTGTTTCGGCCGTTTCTTGGGTGGCTGTACAAGCACTTTGTATA } \\
\text { GTTCATCCATGCCATG }\end{array}$ & $\begin{array}{l}\text { GFP PCR with spop-1 overlaps, } \\
\text { CRISPR repair template }\end{array}$ \\
\hline OG1532 & GAAGATGGAAGCGTTCAACTAGCA & spop-1::gfp genotyping \\
\hline OG1533 & GAGATTCAAGACATTTCGATGAC & spop-1::gfp genotyping \\
\hline OG1819 & GATTTGTACAGGGAAAGGAT & W195G / F197V gRNA \\
\hline OG1820 & $\begin{array}{l}\text { CACAGCGGGCTTATCGATTTGTACAGGGAAAGGATGGAGGATT } \\
\text { CAAAAAATTCATTCGACGAGATTTTTTGTT }\end{array}$ & W195G repair oligo \\
\hline OG1822 & GCCGCGAAGAAATGGGCGAAG & W195G genotyping \\
\hline OG1823 & CCTGTCACGTTTACTGTTTCGGC & W195G genotyping \\
\hline OG1824 & CTGAAATCTTCCACGTTTTCCGCCG & W195G genotyping \\
\hline OG1825 & CGGCTACCACAGACACCTGAAAATTG & W195G genotyping \\
\hline OG1821 & $\begin{array}{l}\text { AGGAGACGAAAGCTATGGAATCACAGCGGGCTTATCGCTTTGT } \\
\text { CCAAGGTAAAGACTGGGGAGTTAAAAAATTCATTCGACGAGATT } \\
\text { TTTTGTTGGATGA }\end{array}$ & F197V repair oligo \\
\hline OG1826 & ATGCGAAACGAGAGGAGACGAAAGCTATG & F197V genotyping \\
\hline OG1827 & AATCTATCTCCAGGAAGAAGCC & F197V genotyping \\
\hline OG1828 & TCACAGCGGGCTTATCGATTTG & F197V genotyping \\
\hline OG1829 & AACAGTCTGGCAATGGACAAAC & F197V genotyping \\
\hline OG1479 & $\begin{array}{l}\text { CCTGGAGGCTTGCTGAAGGCTGTATGCTGTATAGCACCAGCTC } \\
\text { TCAGCAAGTTTTGGCCACTGACTGACTTGCTGAGCTGGTGCTAT } \\
\text { ACAGGACACAAGGCCTGTTACTAGCACTCACATGGAACAAATGG } \\
\text { CCT }\end{array}$ & SPOP miR 1 sense \\
\hline OG1480 & $\begin{array}{l}\text { CCGGAGGCCATTTGTTCCATGTGAGTGCTAGTAACAGGCCTTGT } \\
\text { GTCCTGTATAGCACCAGCTCAGCAAGTCAGTCAGTGGCCAAAA } \\
\text { CTTGCTGAGAGCTGGTGCTATACAGCATACAGCCTTCAGCAAGC } \\
\text { CTCCAGGCCGG }\end{array}$ & SPOP miR antisense \\
\hline OG1481 & $\begin{array}{l}\text { CCTGGAGGCTTGCTGAAGGCTGTATGCTGAGGAGAACTTCACT } \\
\text { ACCTTGAGTTTTGGCCACTGACTGACTCAAGGTAGAAGTTCTCC } \\
\text { TCAGGACACAAGGCCTGTTACTAGCACTCACATGGAACAAATGG } \\
\text { CCT }\end{array}$ & SPOP miR 2 sense \\
\hline OG1482 & $\begin{array}{l}\text { CCGGAGGCCATTTGTTCCATGTGAGTGCTAGTAACAGGCCTTGT } \\
\text { GTCCTGAGGAGAACTTCTACCTTGAGTCAGTCAGTGGCCAAAAC } \\
\text { TCAAGGTAGTGAAGTTCTCCTCAGCATACAGCCTTCAGCAAGCC } \\
\text { TCCAGGCCGG }\end{array}$ & SPOP miR 2 antisense \\
\hline OG1485 & GCTCTTAAGGCTAGAGTACTT & p1006 miR vector sequencing \\
\hline OG1486 & GGAATTGATCCTTATCGATT & p1006 miR vector sequencing \\
\hline
\end{tabular}




\section{References}

1. DeJesus-Hernandez M, et al. (2011) Expanded GGGGCC hexanucleotide repeat in noncoding region of C9ORF72 causes chromosome 9p-linked FTD and ALS. Neuron 72(2):245-256.

2. Renton AE, et al. (2011) A hexanucleotide repeat expansion in C9ORF72 is the cause of chromosome 9p21-linked ALS-FTD. Neuron 72(2):257-268.

3. Balendra R \& Isaacs AM (2018) C9orf72-mediated ALS and FTD: multiple pathways to disease. Nature reviews. Neurology 14(9):544-558.

4. $\quad$ Ling SC, Polymenidou M, \& Cleveland DW (2013) Converging mechanisms in ALS and FTD: disrupted RNA and protein homeostasis. Neuron 79(3):416-438.

5. Schipper LJ, et al. (2016) Prevalence of brain and spinal cord inclusions, including dipeptide repeat proteins, in patients with the C9ORF72 hexanucleotide repeat expansion: a systematic neuropathological review. Neuropathology and applied neurobiology 42(6):547-560.

6. Davidson YS, et al. (2014) Brain distribution of dipeptide repeat proteins in frontotemporal lobar degeneration and motor neurone disease associated with expansions in C9ORF72. Acta Neuropathol Commun 2:70.

7. Wen X, et al. (2014) Antisense proline-arginine RAN dipeptides linked to C9ORF72-ALS/FTD form toxic nuclear aggregates that initiate in vitro and in vivo neuronal death. Neuron 84(6):1213-1225.

8. Mizielinska S, et al. (2014) C9orf72 repeat expansions cause neurodegeneration in Drosophila through arginine-rich proteins. Science 345(6201):1192-1194.

9. Jovicic A, et al. (2015) Modifiers of C9orf72 dipeptide repeat toxicity connect nucleocytoplasmic transport defects to FTD/ALS. Nature neuroscience 18(9):1226-1229.

10. Zhang $\mathrm{K}$, et al. (2015) The C9orf72 repeat expansion disrupts nucleocytoplasmic transport. Nature 525(7567):56-61.

11. Freibaum BD, et al. (2015) GGGGCC repeat expansion in C9orf72 compromises nucleocytoplasmic transport. Nature 525(7567):129-133.

12. Zhang $\mathrm{YJ}$, et al. (2018) Poly(GR) impairs protein translation and stress granule dynamics in C9orf72-associated frontotemporal dementia and amyotrophic lateral sclerosis. Nature medicine 24(8):1136-1142.

13. Guo Q, et al. (2018) In Situ Structure of Neuronal C9orf72 Poly-GA Aggregates Reveals Proteasome Recruitment. Cell 172(4):696-705 e612.

14. Gupta R, et al. (2017) The Proline/Arginine Dipeptide from Hexanucleotide Repeat Expanded C9ORF72 Inhibits the Proteasome. eNeuro 4(1).

15. Yin S, et al. (2017) Evidence that C9ORF72 Dipeptide Repeat Proteins Associate with U2 snRNP to Cause Mis-splicing in ALS/FTD Patients. Cell reports 19(11):2244-2256.

16. Choi SY, et al. (2019) C9ORF72-ALS/FTD-associated poly(GR) binds Atp5a1 and compromises mitochondrial function in vivo. Nature neuroscience 22(6):851-862. 
17. Boeynaems S, et al. (2017) Phase Separation of C9orf72 Dipeptide Repeats Perturbs Stress Granule Dynamics. Molecular cell 65(6):10441055 e1045.

18. Lee $\mathrm{KH}$, et al. (2016) C9orf72 Dipeptide Repeats Impair the Assembly, Dynamics, and Function of Membrane-Less Organelles. Cell 167(3):774788 e717.

19. Kramer NJ, et al. (2018) CRISPR-Cas9 screens in human cells and primary neurons identify modifiers of C9ORF72 dipeptide-repeat-protein toxicity. Nature genetics 50(4):603-612.

20. Rudich P, et al. (2017) Nuclear localized C9orf72-associated argininecontaining dipeptides exhibit age-dependent toxicity in C. elegans. Hum Mol Genet 26(24):4916-4928.

21. Boeynaems S, et al. (2016) Drosophila screen connects nuclear transport genes to DPR pathology in c9ALS/FTD. Sci Rep 6:20877.

22. Lin Y, et al. (2016) Toxic PR Poly-Dipeptides Encoded by the C9orf72 Repeat Expansion Target LC Domain Polymers. Cell 167(3):789-802 e712.

23. Ohki Y, et al. (2017) Glycine-alanine dipeptide repeat protein contributes to toxicity in a zebrafish model of C9orf72 associated neurodegeneration. Molecular neurodegeneration 12(1):6.

24. Farg MA, et al. (2017) C9ORF72, implicated in amytrophic lateral sclerosis and frontotemporal dementia, regulates endosomal trafficking. Human molecular genetics 26(20):4093-4094.

25. Farg MA, Konopka A, Soo KY, Ito D, \& Atkin JD (2017) The DNA damage response (DDR) is induced by the C9orf72 repeat expansion in amyotrophic lateral sclerosis. Human molecular genetics 26(15):2882-2896.

26. Clark A \& Burleson M (2020) SPOP and cancer: a systematic review. Am J Cancer Res 10(3):704-726.

27. Chai N \& Gitler AD (2018) Yeast screen for modifiers of C9orf72 poly(glycine-arginine) dipeptide repeat toxicity. FEMS Yeast Res 18(4).

28. Fischer SE, et al. (2013) Multiple small RNA pathways regulate the silencing of repeated and foreign genes in C. elegans. Genes Dev 27(24):2678-2695.

29. Jablonski AM, et al. (2015) Loss of RAD-23 Protects Against Models of Motor Neuron Disease by Enhancing Mutant Protein Clearance. The Journal of neuroscience : the official journal of the Society for Neuroscience 35(42):14286-14306.

30. Zhai J, et al. (2015) Inhibition of Cytohesins Protects against Genetic Models of Motor Neuron Disease. The Journal of neuroscience : the official journal of the Society for Neuroscience 35(24):9088-9105.

31. Li G, et al. (2014) SPOP promotes tumorigenesis by acting as a key regulatory hub in kidney cancer. Cancer Cell 25(4):455-468.

32. Guo ZQ, et al. (2016) Small-Molecule Targeting of E3 Ligase Adaptor SPOP in Kidney Cancer. Cancer Cell 30(3):474-484.

33. Kwon I, et al. (2014) Poly-dipeptides encoded by the C9orf72 repeats bind nucleoli, impede RNA biogenesis, and kill cells. Science 345(6201):11391145. 
34. Nagai $Y$, et al. (1997) Identification of a novel nuclear speckle-type protein, SPOP. FEBS letters 418(1-2):23-26.

35. Marzahn MR, et al. (2016) Higher-order oligomerization promotes localization of SPOP to liquid nuclear speckles. The EMBO journal 35(12):1254-1275.

36. Bouchard JJ, et al. (2018) Cancer Mutations of the Tumor Suppressor SPOP Disrupt the Formation of Active, Phase-Separated Compartments. Molecular cell 72(1):19-36 e18.

37. White MR, et al. (2019) C9orf72 Poly(PR) Dipeptide Repeats Disturb Biomolecular Phase Separation and Disrupt Nucleolar Function. Molecular cell 74(4):713-728 e716.

38. Geng C, et al. (2013) Prostate cancer-associated mutations in speckle-type POZ protein (SPOP) regulate steroid receptor coactivator 3 protein turnover. Proceedings of the National Academy of Sciences of the United States of America 110(17):6997-7002.

39. Barbieri CE, et al. (2012) Exome sequencing identifies recurrent SPOP, FOXA1 and MED12 mutations in prostate cancer. Nature genetics 44(6):685-689.

40. Blattner $\mathrm{M}$, et al. (2014) SPOP mutations in prostate cancer across demographically diverse patient cohorts. Neoplasia 16(1):14-20.

41. Blattner M, et al. (2017) SPOP Mutation Drives Prostate Tumorigenesis In Vivo through Coordinate Regulation of $\mathrm{PI} K \mathrm{~K} / \mathrm{mTOR}$ and $\mathrm{AR}$ Signaling. Cancer Cell 31(3):436-451.

42. Dai X, et al. (2017) Prostate cancer-associated SPOP mutations confer resistance to BET inhibitors through stabilization of BRD4. Nature medicine 23(9):1063-1071.

43. Janouskova $\mathrm{H}$, et al. (2017) Opposing effects of cancer-type-specific SPOP mutants on BET protein degradation and sensitivity to BET inhibitors. Nature medicine 23(9):1046-1054.

44. Theurillat JP, et al. (2014) Prostate cancer. Ubiquitylome analysis identifies dysregulation of effector substrates in SPOP-mutant prostate cancer. Science 346(6205):85-89.

45. Ma J, et al. (2018) SPOP promotes ATF2 ubiquitination and degradation to suppress prostate cancer progression. J Exp Clin Cancer Res 37(1):145.

46. Zhang $P$, et al. (2017) Intrinsic BET inhibitor resistance in SPOP-mutated prostate cancer is mediated by BET protein stabilization and AKT-mTORC1 activation. Nature medicine 23(9):1055-1062.

47. Fujisawa $T$ \& Filippakopoulos $P$ (2017) Functions of bromodomaincontaining proteins and their roles in homeostasis and cancer. Nat Rev Mol Cell Biol 18(4):246-262.

48. Corman A, et al. (2019) A Chemical Screen Identifies Compounds Limiting the Toxicity of C9ORF72 Dipeptide Repeats. Cell Chem Biol 26(2):235-243 e235.

49. Dong Z, et al. (2020) Structure-Activity Relationship of SPOP Inhibitors against Kidney Cancer. Journal of medicinal chemistry 63(9):4849-4866. 
50. Rohlfing AK, Miteva Y, Moronetti L, He L, \& Lamitina T (2011) The Caenorhabditis elegans mucin-like protein OSM-8 negatively regulates osmosensitive physiology via the transmembrane protein PTR-23. PLoS genetics 7(1):e1001267.

51. Lamitina T, Huang CG, \& Strange K (2006) Genome-wide RNAi screening identifies protein damage as a regulator of osmoprotective gene expression. Proceedings of the National Academy of Sciences of the United States of America 103(32):12173-12178.

52. Ghanta KS \& Mello CC (2020) Melting dsDNA Donor Molecules Greatly Improves Precision Genome Editing in Caenorhabditis elegans. Genetics 216(3):643-650.

53. Rudich P, Snoznik, C., Puleo, N., Lamitina, T. (2020) Methods for measuring RAN peptide toxicity in C. elegans. Journal of Visualized Experiments In Press.

54. McGurk L, et al. (2018) Poly(ADP-Ribose) Prevents Pathological Phase Separation of TDP-43 by Promoting Liquid Demixing and Stress Granule Localization. Molecular cell 71(5):703-717 e709.

55. Gupta P, Uner OE, Nayak S, Grant GR, \& Kalb RG (2018) SAP97 regulates behavior and expression of schizophrenia risk enriched gene sets in mouse hippocampus. PloS one 13(7):e0200477.

56. Mojsilovic-Petrovic J, et al. (2006) Protecting motor neurons from toxic insult by antagonism of adenosine A2a and Trk receptors. The Journal of neuroscience : the official journal of the Society for Neuroscience 26(36):9250-9263.

57. Han SK, et al. (2016) OASIS 2: online application for survival analysis 2 with features for the analysis of maximal lifespan and healthspan in aging research. Oncotarget.

58. Yang JS, et al. (2011) OASIS: online application for the survival analysis of lifespan assays performed in aging research. PloS one 6(8):e23525.

59. Shibata Y, Takeshita H, Sasakawa N, \& Sawa H (2010) Double bromodomain protein BET-1 and MYST HATs establish and maintain stable cell fates in C. elegans. Development 137(7):1045-1053.

60. Rudich P, Snoznik C, Puleo N, \& Lamitina T (2020) Measuring RAN Peptide Toxicity in C. elegans. $J$ Vis Exp (158).

61. Thompson O, et al. (2013) The million mutation project: a new approach to genetics in Caenorhabditis elegans. Genome Res 23(10):1749-1762. 


\section{Figure 1}

A
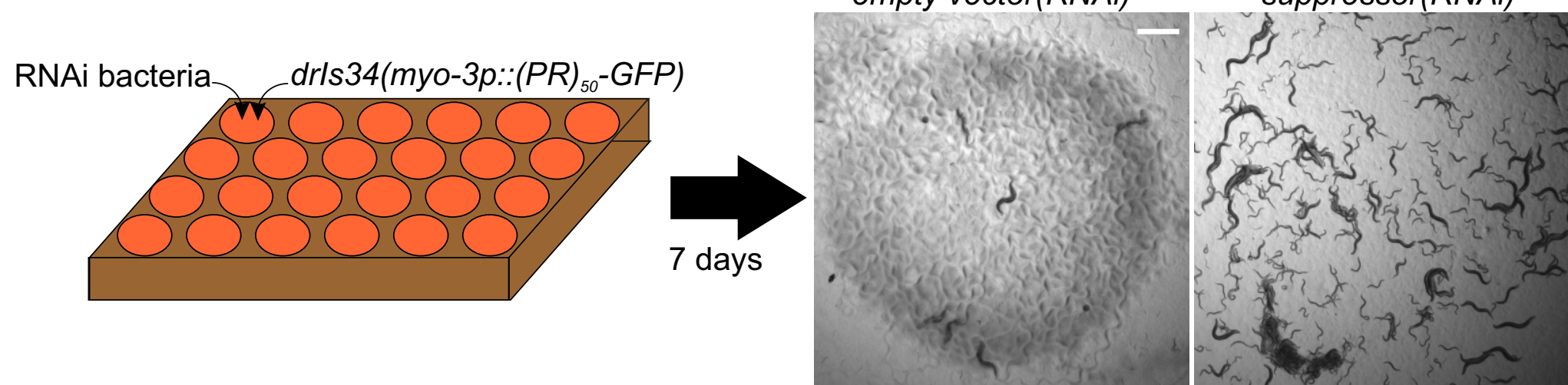

B
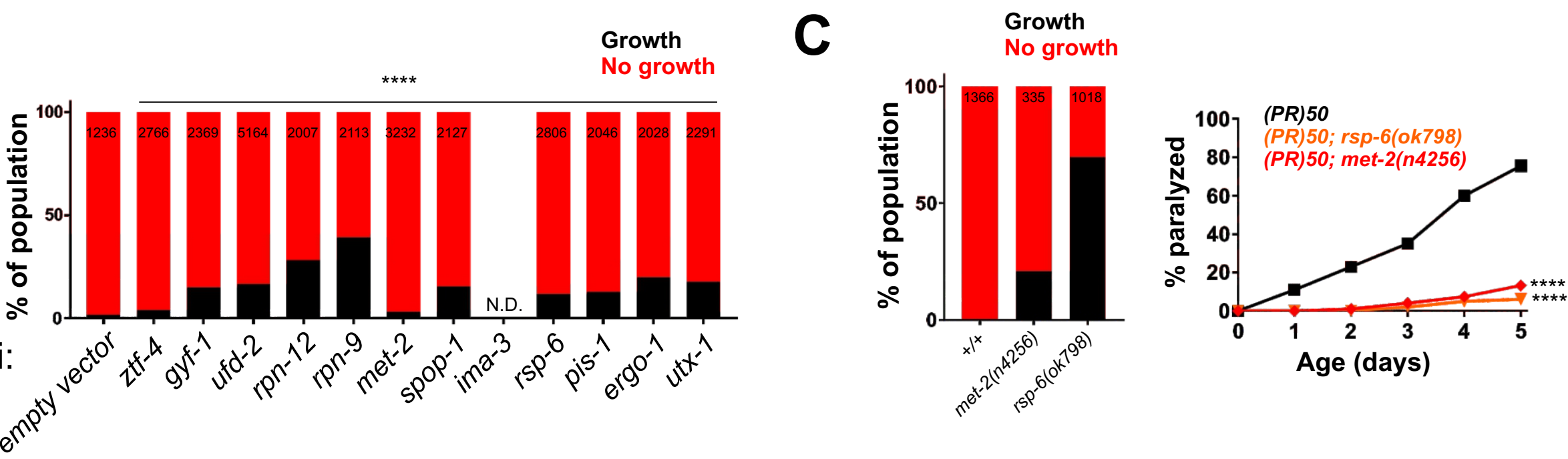

D

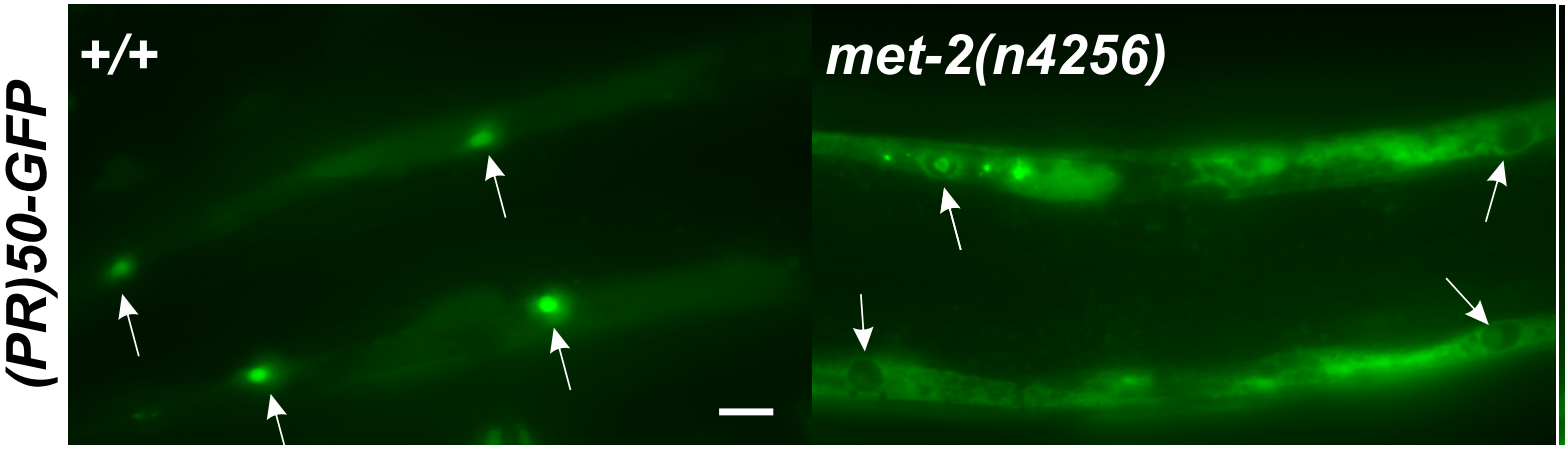




\section{A \\ C}
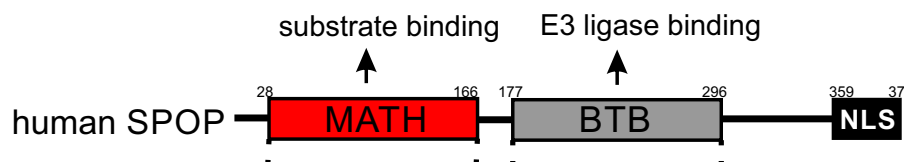

C. elegans spop-1

$\%$ identity:

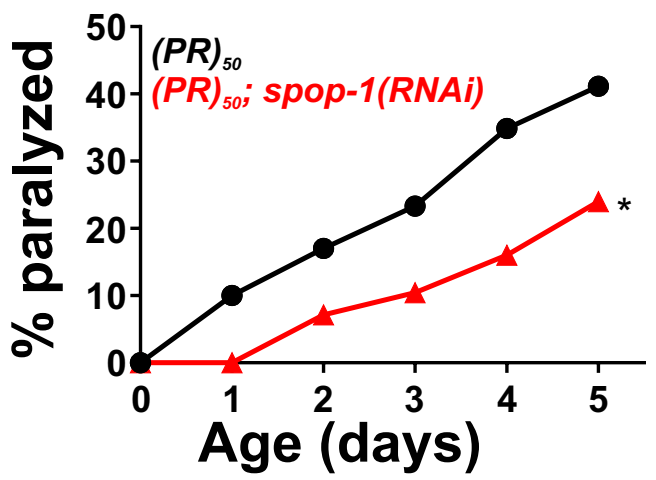

$\mathbf{E}$

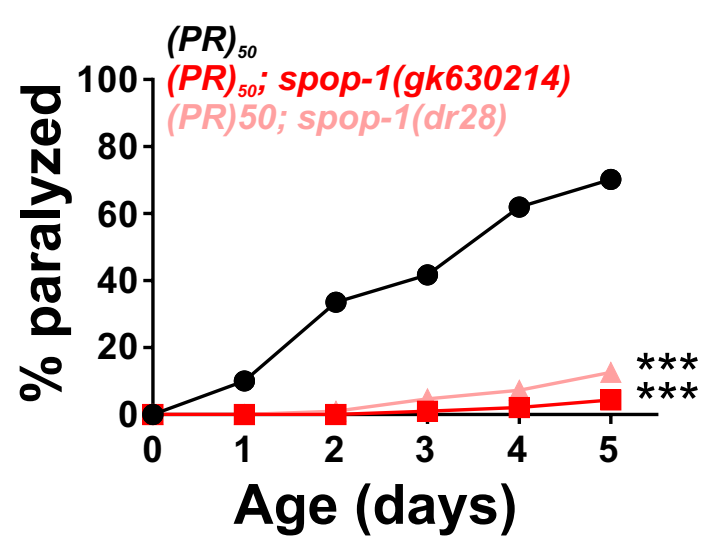

$\mathbf{F}$
B

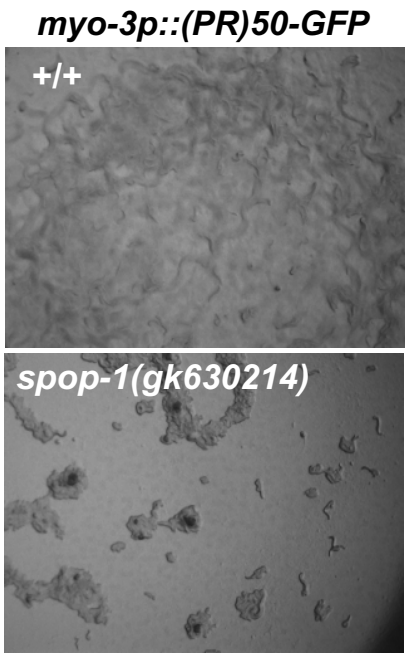

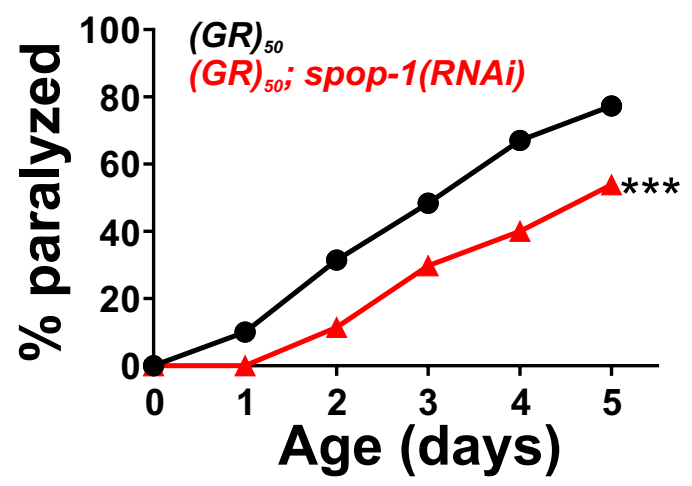

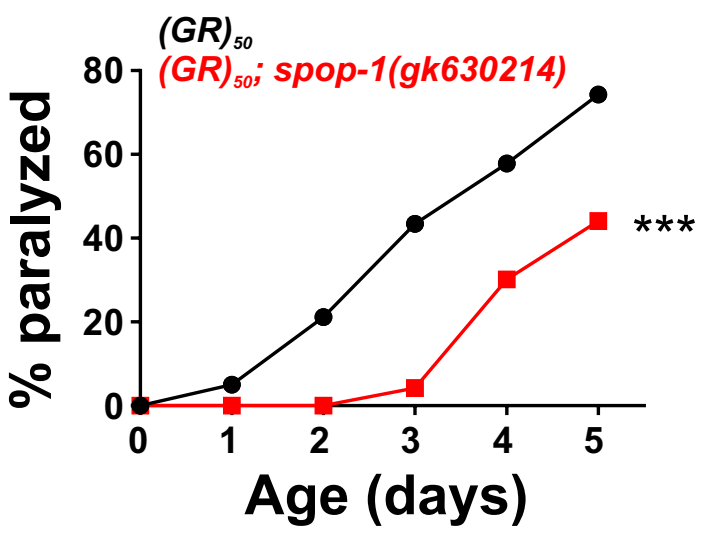




\section{Figure 3}

A
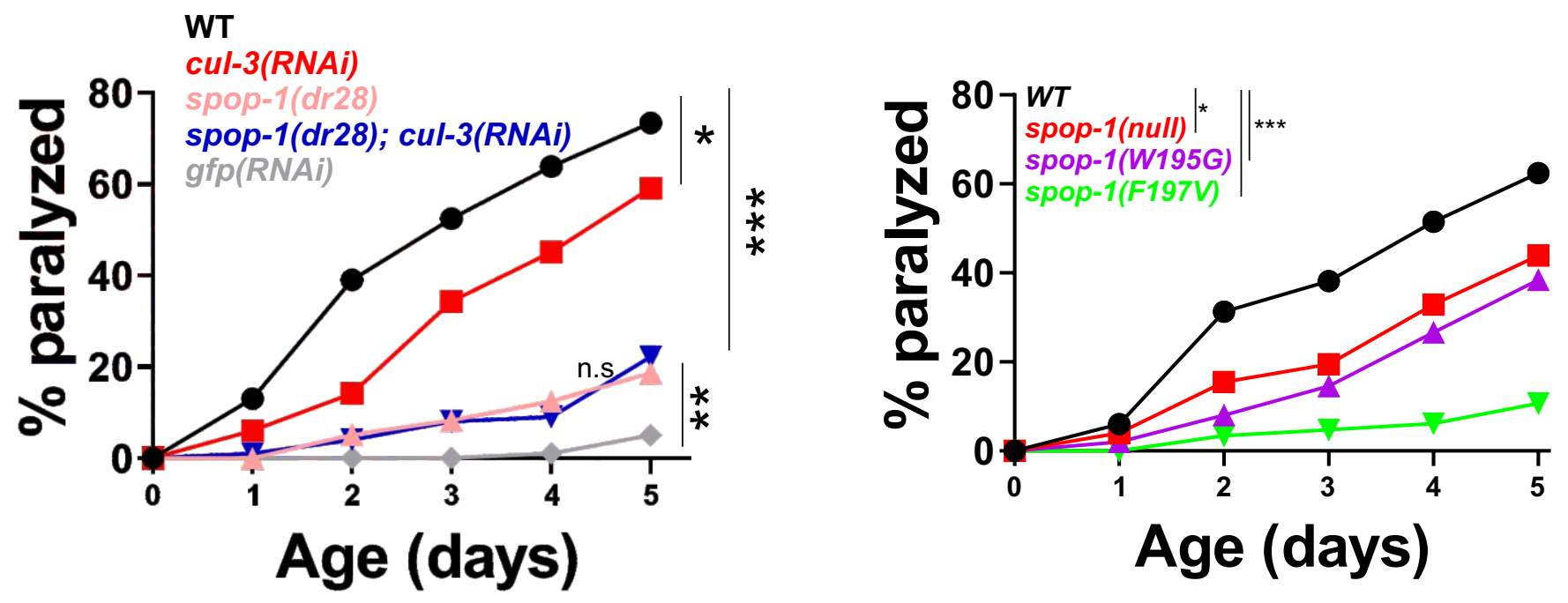

C

SPOP-1::GFP
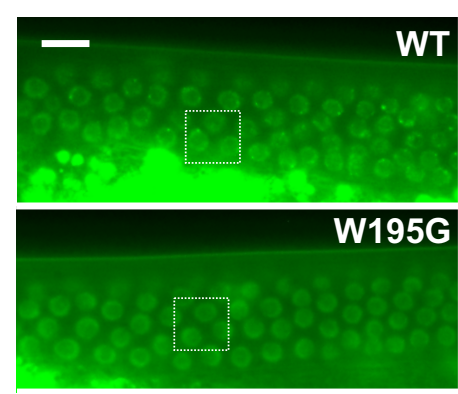

F197V
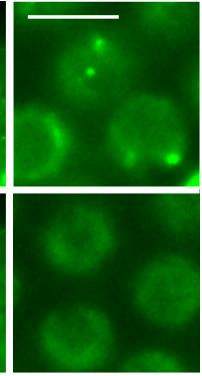

D
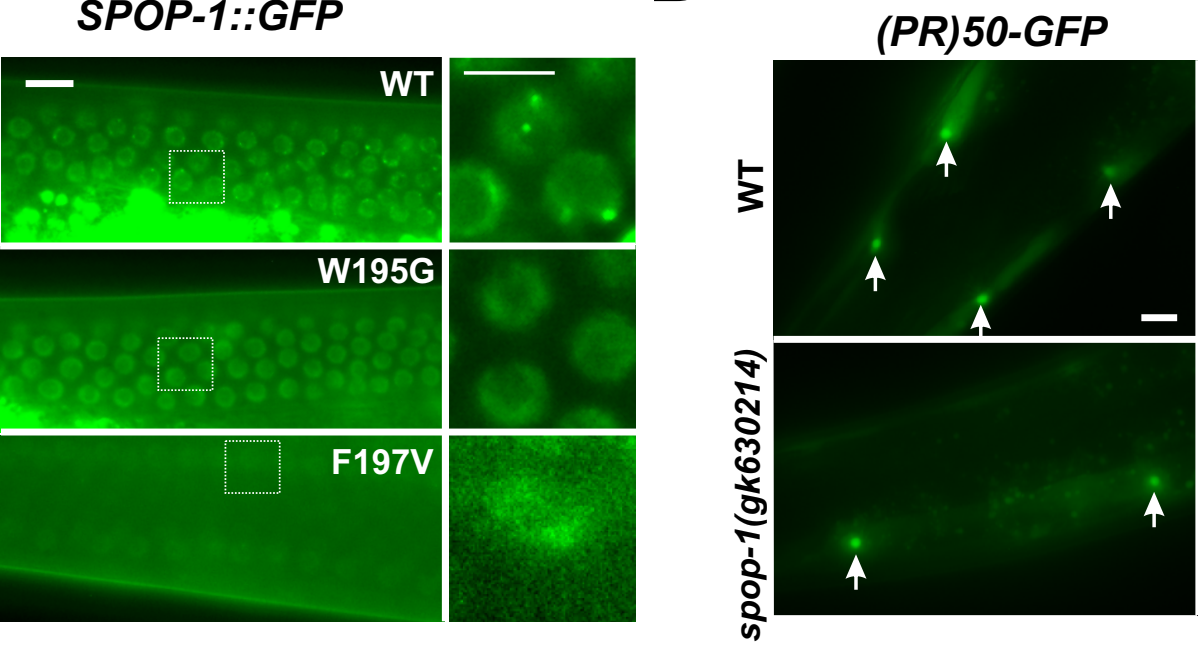

(GR)50-GFP

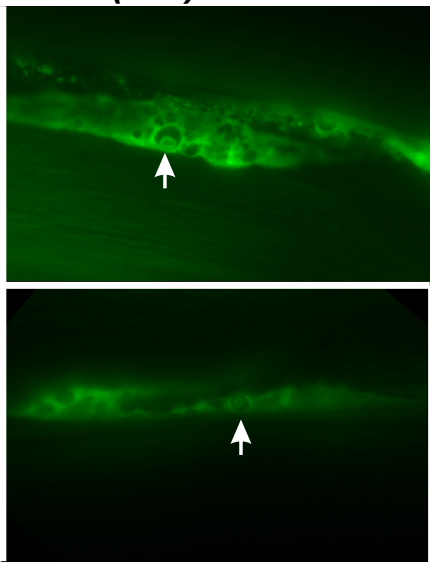




\section{Figure 4}

A

Primary cortical neurons

$-\quad+\quad-\quad-$ LacZ

$+\quad+\quad-\quad$ Scr miRNA

- $\quad-\quad+\quad-$ SPOP MIRNA\#1

- $\quad$ - $\quad$ - $\quad+$ SPOP miRNA \#2

$-1=\overline{\text { Anti-SPOP }}$

Anti-TUBB3

C SPOP substrates

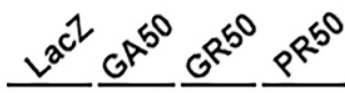

$\longrightarrow-\infty-\infty-P T E N$

$-\infty=-\infty=0$ DUSP7

$-\infty-\infty-63 B P 1$

$-\infty-\infty-\infty$ TUBB3

$-+-++_{-}++$miRNA

B

to SPOP
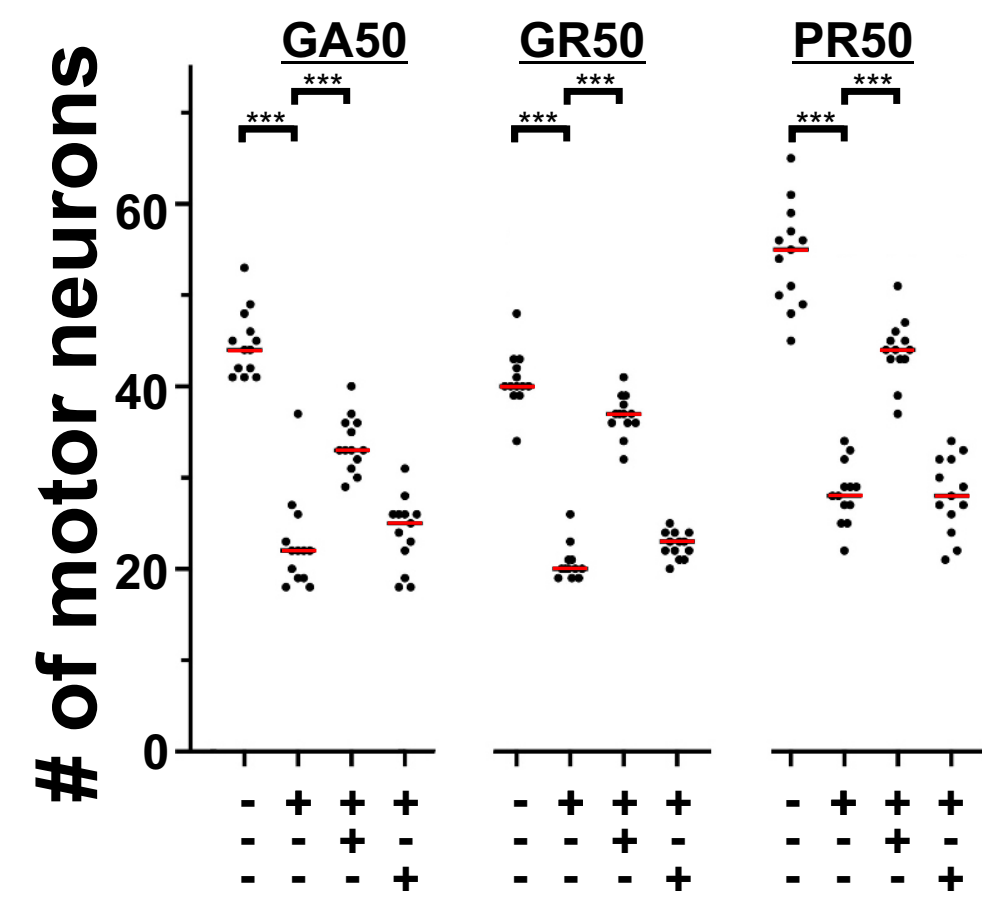

D

GA50

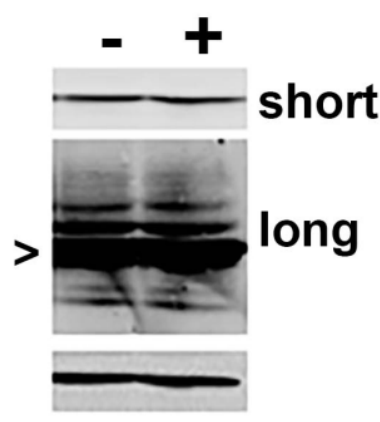

GR50

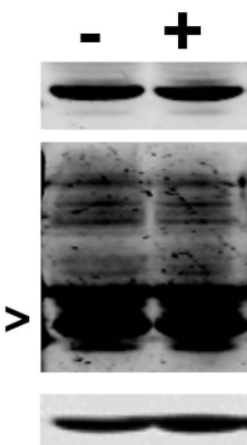

PR50

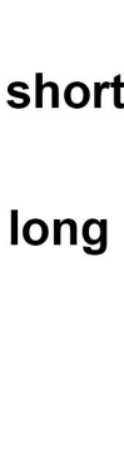

active SPOP miRNA
DPR

SPOP miR control miR short

long

anti-Tubulin 


\section{Figure 5}
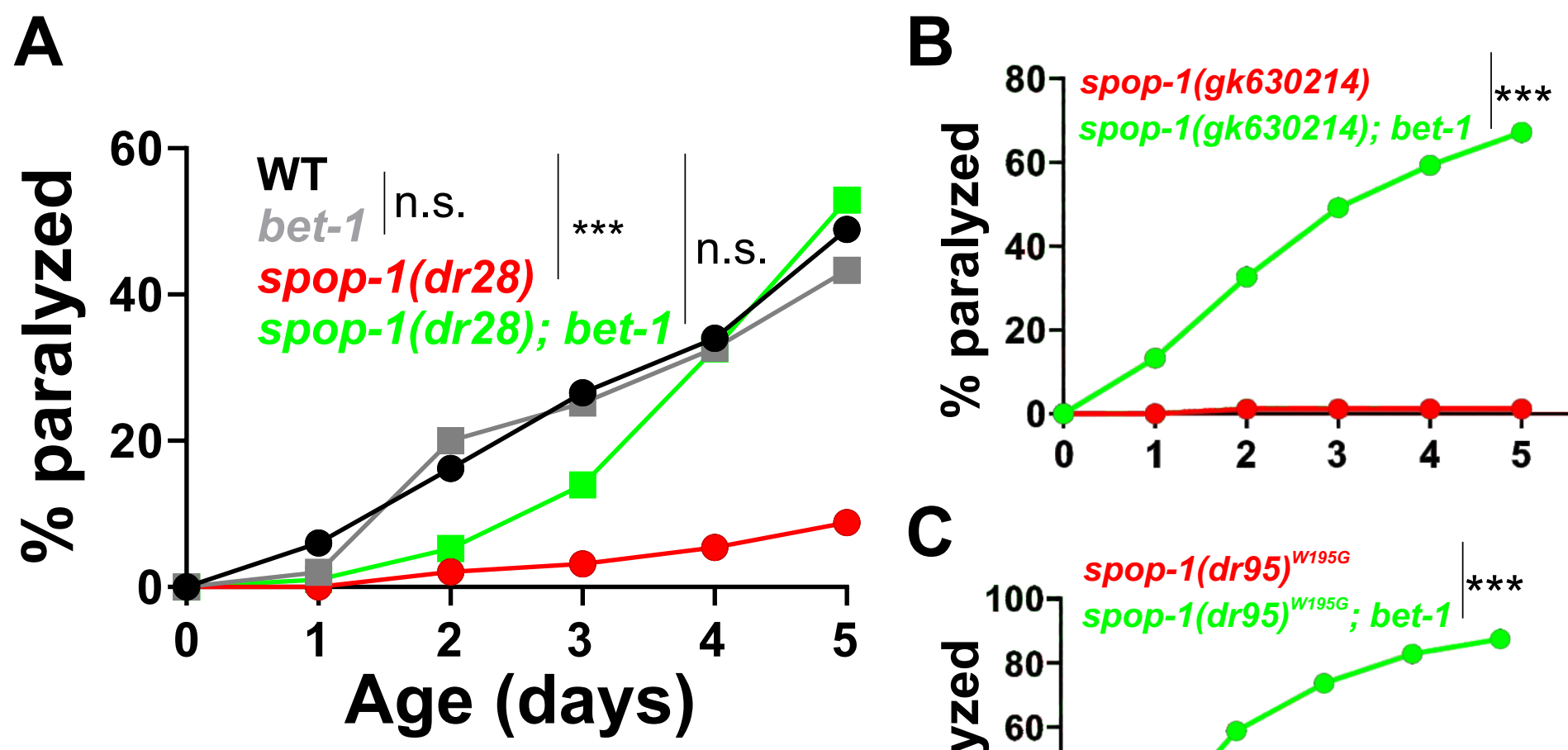

D

C

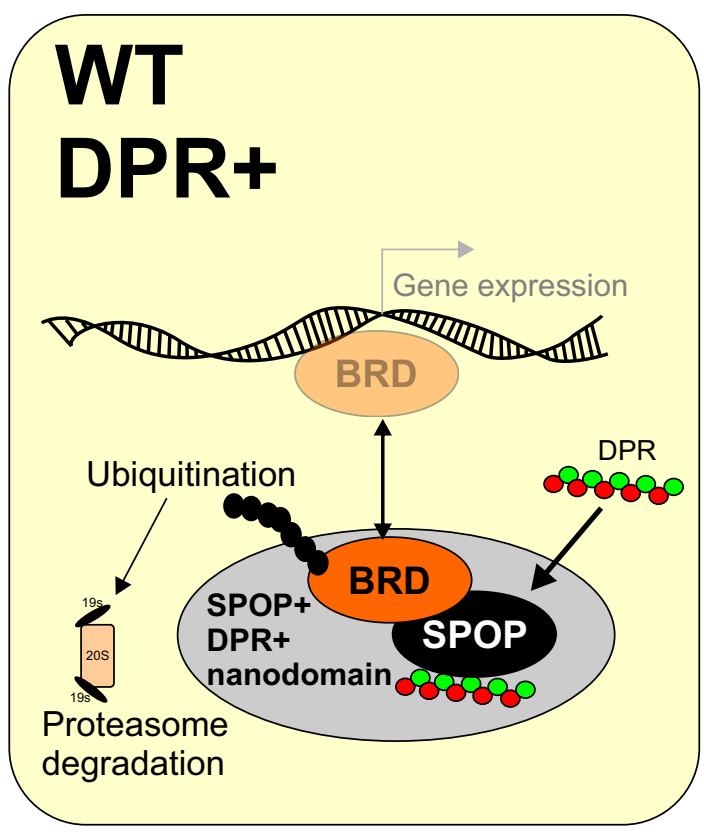

Neurotoxic

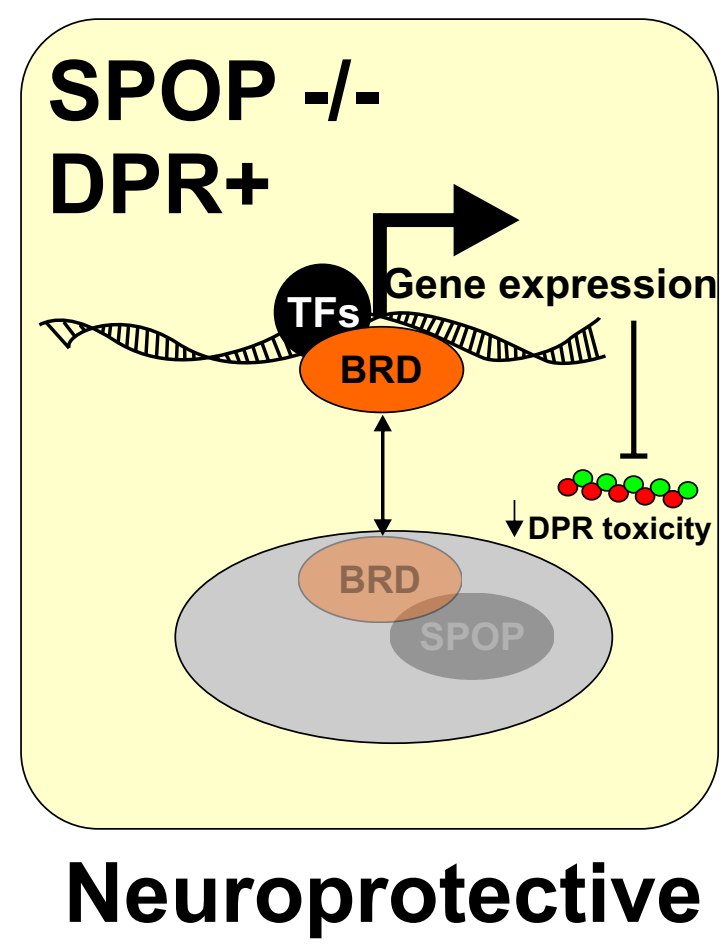




\section{Figure S1}

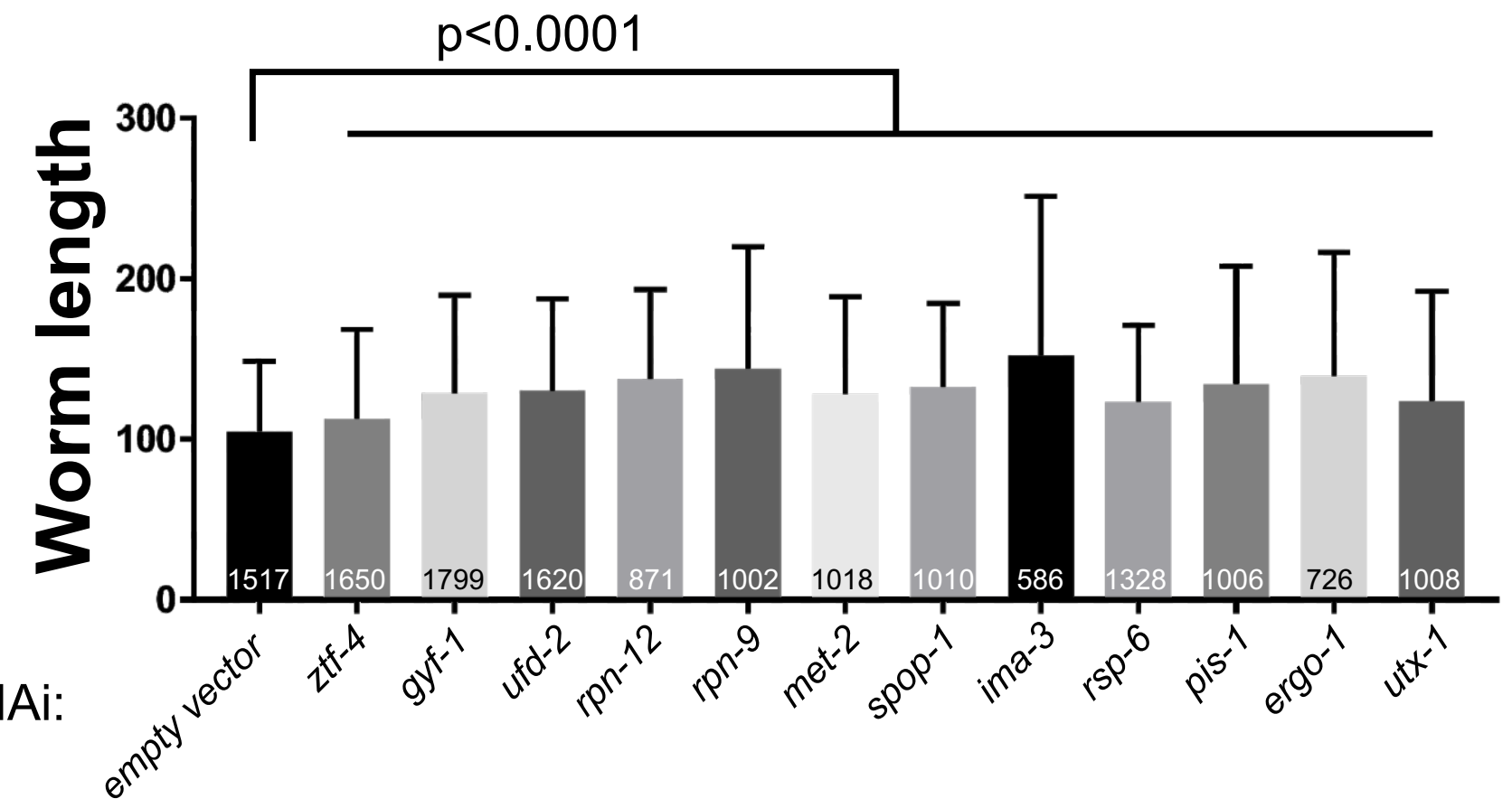


ztf-4(RNAi)

gyf-1(RNAi)

ufd-2(RNAi)

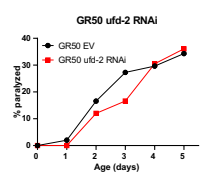

rpn-12(RNAi)

$r p n-9(R N A i)$

met-2(RNAi)

spop-1(RNAi)

ima-3(RNAi)

$r s p-6(R N A i)$

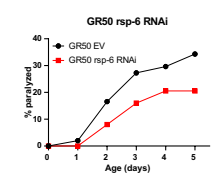

pis-1(RNAi)

ergo-1(RNAi)

$u t x-1(R N A i)$
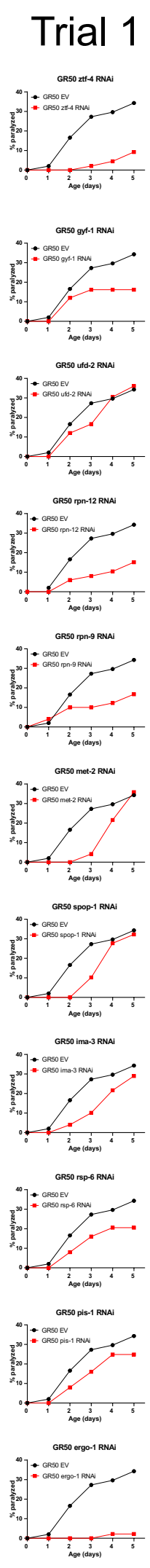

\section{Trial 2}
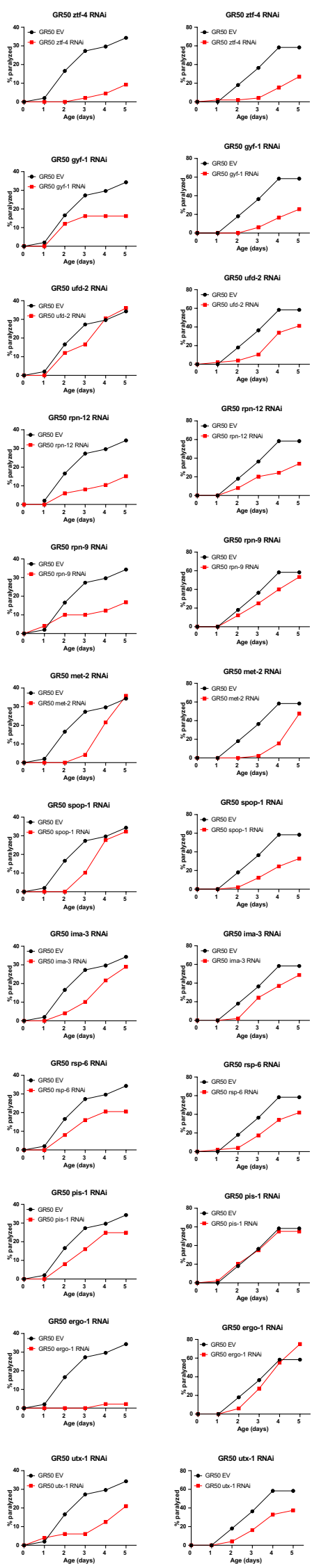

\# of trials with Log-rank significant $p$-value

$2 / 2 p<0.05$

$1 / 2 p<0.05$

$1 / 2 p<0.05$

$1 / 2 p<0.05$

$1 / 2 p<0.05$

$1 / 2 p<0.05$

$1 / 2 p<0.05$

$0 / 2 p<0.05$

$1 / 2 p<0.05$

$0 / 2 p<0.05$

$1 / 2 p<0.05$

$1 / 2 p<0.05$ 


\section{Figure S3}

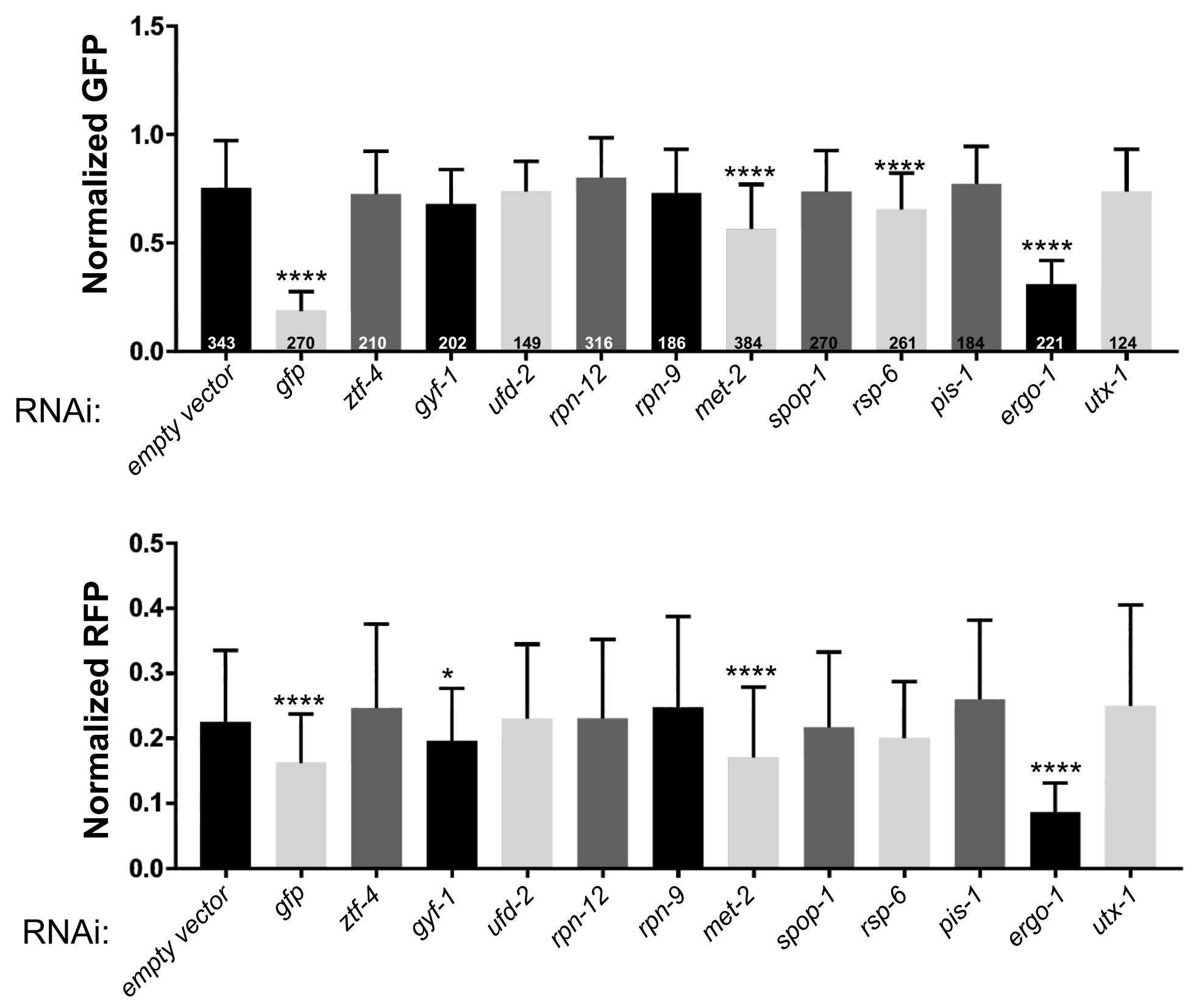


Figure S4

\section{Motor neuron (GR)50 expression}

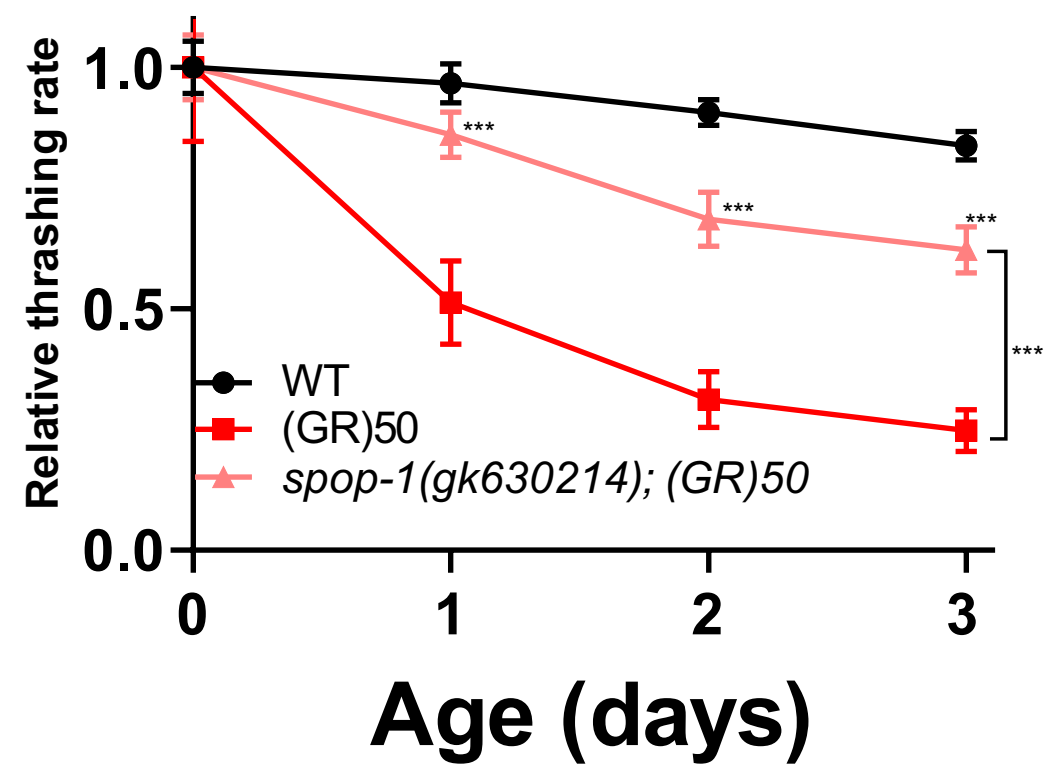




\section{Figure S5}

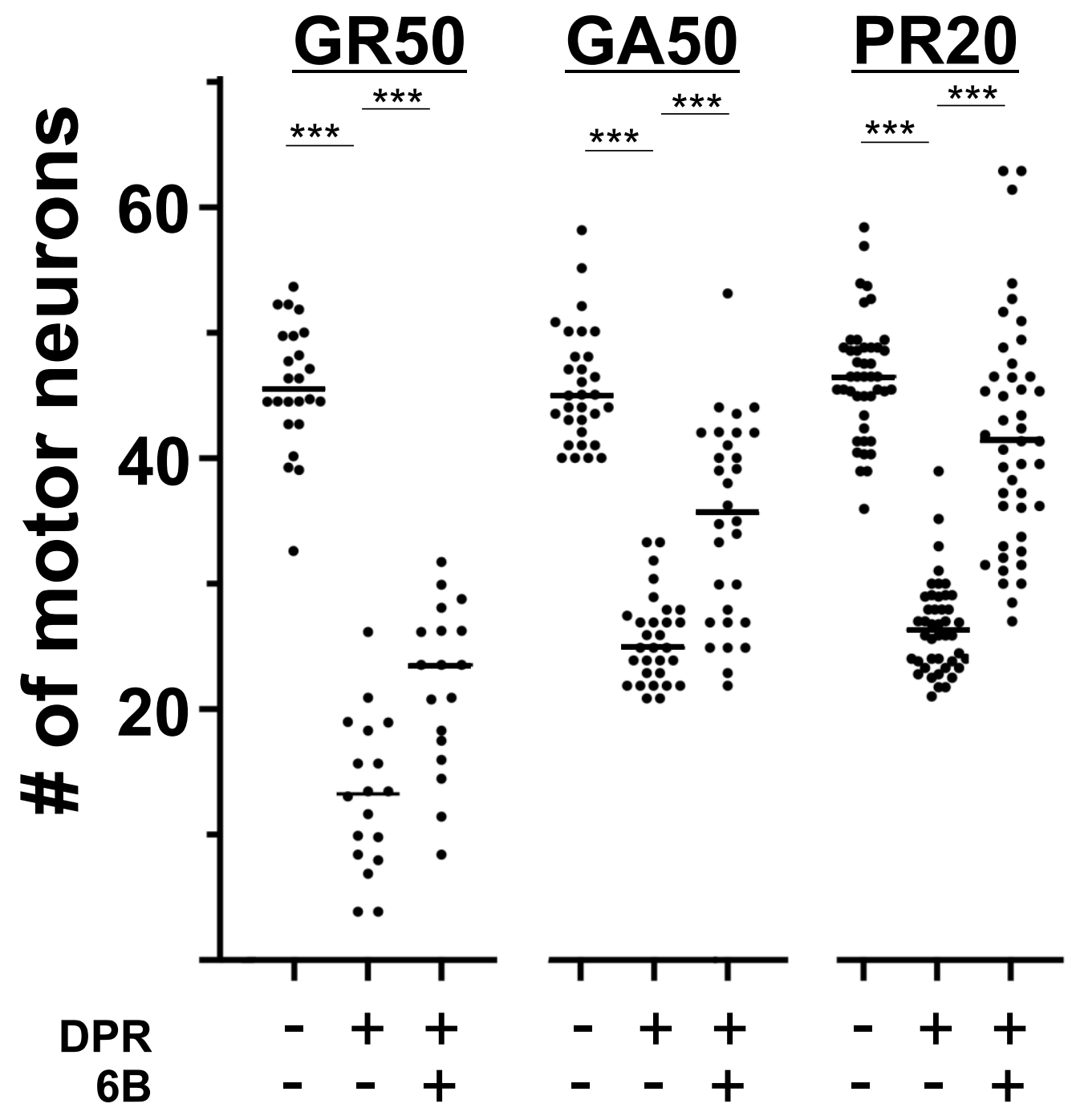

